\title{
Machtwille und Machtressourcen der lateinamerikanischen Parlamente
}

\author{
Unter besonderer Berücksichtigung des argentinischen Kongresses, \\ seiner ,technischen Krise“ 1999 und der Ereignisse Anfang 2002
}

Von Agustín Ferraro, Buenos Aires

\section{Einführung}

Das argentinische Parlament ${ }^{1}$ ist 1999 in eine schwere technische Krise geraten, als es versuchte, alle auf die Exekutive übertragenen Kompetenzen zur Rechtsetzung zurück zu fordern. Nach der 1994 reformierten Verfassung muss das argentinische Parlament alle gesetzgeberische Gewalt als seine eigentliche Kompetenz wahrnehmen. Der Präsident hat sich mit nur wenigen Ausnahmen auf seine Rolle als ausführendes Organ zu beschränken. Mit anderen Worten: Die legislative Gewalt auf die Exekutive zu delegieren, war verboten. Die reformierte Verfassung gab dem Kongress eine Frist von fünf Jahren, nach deren Ablauf das (gewiss drakonische) Verbot in die Praxis umgesetzt werden sollte. Als der Zeitpunkt näherrückte (25. August 1999), war dies jedoch aus verschiedenen Gründen technisch unmöglich. Im Bewusstsein seiner technischen Unfähigkeit begann der Kongress rasch mit den nötigen Arbeiten, um in drei Jahren (bis 2002) endgültig alle an die Exekutive übertragenen Kompetenzen zur Rechtsetzung wieder selber wahrnehmen zu können.

Da diese parlamentarische Krise juristisch-technischer und nicht unmittelbar politischer Natur gewesen ist, fand sie hauptsächlich Resonanz in der juristischen Literatur und teilweise in den Medien. Sie ließ indes immerhin eine wichtige politische Tatsache erkennen: Der politische Prozess in Argentinien - wie übrigens auch in anderen lateinamerikanischen Ländern - muss keinesfalls so verstanden werden, als engagierten sich alle relevanten Akteure für die Erhaltung und sogar Erweiterung einer präsidialen Dominanz. Die gängige Vorstellung von der Allmacht des Präsidenten gegenüber ohnmächtigen Parlamenten in Lateinamerika lässt sich aus verschiedenen Gründen immer weniger aufrechterhalten. Deshalb werden im folgenden zunächst die 1999er Krise des argentinischen Parlaments wie die Chancen für ihre Bewältigung bis zum Ablauf der nächsten Frist im Jahre 2002 diskutiert (1). 
Wie anschließend aufgezeigt werden soll (2), sind die Parlamente ein oft unterschätzter Machtfaktor in der lateinamerikanischen Politik. Sie besitzen beachtliche Machtressourcen. Hier wird für Argentinien - wenngleich nur in Form eines Exkurses (3) - auch auf die Regierungskrise im Dezember 2001 / Januar 2002 einzugehen sein, um die politische Macht eines lateinamerikanischen Parlaments anhand eines offenen Machtkampfes mit der Exekutive zu illustrieren.

Sodann (4) wird den Ursachen für die Erfolglosigkeit mancher Reformen im Parlamentssystem lateinamerikanischer Staaten nachgegangen. Sie liegt weniger in der Unentschlossenheit der relevanten Akteure, als eher in einem - manchmal auch nur unbewussten Mangel an Kenntnissen und Expertise über die Instrumente der Parlamentsarbeit. Dabei spielt gewiss der relativ kurze Erfahrungshintergrund vieler lateinamerikanischer Demokratien (weniger als 20 Jahre in Argentinien, Brasilien, Chile, Uruguay etc.) eine Rolle. Es gibt aber zusätzliche Gründe für die mangelnde Professionalität von Parlamentariern. In diesem Zusammenhang geboten erscheint eine vergleichende Perspektive der Instrumente parlamentarischer Arbeit, die sehr wohl als parlamentarische Machtressourcen gebraucht werden können. Daher sollen abschließend drei Instrumente der Parlamentsarbeit dargestellt werden, die jeweils für die USA, Großbritannien und Deutschland charakteristisch sind (5). Mit zwei von diesen Instrumenten wurde ansatzweise auch in Lateinamerika experimentiert. Versuche und Experimente dieser Art sind ohne Zweifel unentbehrlich, um eine realistische Bewertungsgrundlage für eine Verbesserung und Stärkung der Rolle der lateinamerikanischen Parlamente zu gewinnen.

\section{Die technische Krise des argentinischen Parlaments im Jahr 1999}

Ein erklärtes Ziel der argentinischen Verfassungsreform von 1994 war es, die politische Rolle des Kongresses gegenüber der Exekutive zu stärken. Die Begrenzung der präsidialen Macht wurde zwischen dem damaligen Präsidenten Menem und der Opposition als Ausgleich dafür ausgehandelt, dass im Gegenzug die reformierte Verfassung eine Wiederwahl des Präsidenten zulassen sollte. Bloßes Machtkalkül war jedoch nicht der einzige Anlass für diesen Kompromiss. Für die Opposition war die Begrenzung der präsidialen Macht in mancher Hinsicht eine Prinzipienfrage, zumal die angestrebten Reformen die präsidiale Macht auch im Falle eines Machtwechsels einschränken würden.

Nicht nur die damalige Opposition, sondern auch die Regierungspartei standen bei dieser Frage maßgeblich unter dem Einfluss der Debatte über den (lateinamerikanischen) Präsidentialismus, einer Kontroverse, die Mitte der achtziger Jahre die Arbeiten des amerikani- 
schen Politologen Juan Linz entfacht hatten. ${ }^{2}$ Die Machtkonzentration im Amt des Präsidenten, so behauptete Linz, sei die wesentliche Ursache für den Mangel an politischer Stabilität in den lateinamerikanischen Demokratien. Für eine Stabilisierung dieser Demokratien wäre nach Meinung von Linz eine Reform ihrer Verfassungsstrukturen nötig, welche das bestehende Präsidialsystem in ein parlamentarisches System europäischer Prägung verwandeln würde. Im Einklang mit Linz wurden der damaligen argentinischen Präsidialverfassung diverse parlamentarische Elemente hinzugefügt, wie beispielsweise die Figur eines gegenüber dem Parlament verantwortlichen Kabinettschefs und andere Bestimmungen, die den politischen Einfluss des Präsidenten zugunsten des Parlaments eingrenzen sollten.

Jede Verfassung versucht nun ein gewisses Modell der Machtverteilung gegen künftige politische Konjunkturen abzusichern. Die beabsichtigte Stärkung der parlamentarischen Macht hätte sich in diesem Sinne als völlig wirkungslos erweisen können, sobald ein künftiges Parlament, in dem die Regierungspartei über eine starke Parlamentsmehrheit und fügsame Parlamentsmitglieder verfügt, dem Präsidenten besondere Vorrechte einräumt. Gegen solche Kontingenzen wollte insbesondere die Reform des Artikels 76 die Verfassung absichern. In diesem Artikel wird dem Kongress die Delegation gesetzgeberischer Gewalt an die Exekutive einfach verboten. ${ }^{3}$ Die Rolle des Kongresses als Gesetzgeber, d.h.

Die Diskussion begann 1984 mit verschiedenen Versionen eines Manuskripts von Linz, das große Verbreitung unter Spezialisten in den USA, Lateinamerika und Europa fand. Eine Zusammenfassung seiner Ideen wurde von Linz 1988 auf Spanisch in Argentinien veröffentlicht: Juan Linz, Democracia Presidencialista o Parlamentaria ¿Hay alguna diferencia?, in: Consejo para la Consolidación de la Democracia (Hrsg.), Presidencialismo vs. Parlamentarismo: Materiales para el estudio de la Reforma Constitucional, Buenos Aires: Eudeba, 1988, S. 19-43. Diese Publikation hatte jedoch nur regionale Zirkulation. Als Standard der wissenschaftlichen Literatur gelten deswegen eher Juan Linz, The Perils of Presidentialism, Journal of Democracy, Bd. 1 (1), 1990, S. 51-69, und Juan Linz, The Virtues of Parliamentarism, Journal of Democracy, Bd. 1 (4), 1990, S. 84-91. Das originale Manuskript wurde wohlgemerkt erst 1994 in vollem Umfang veröffentlicht: Juan Linz, Presidential or Parliamentary Democracy: Does It Make a Difference?, in: Juan Linz / Arturo Valenzuela (Hrsg.), The Failure of Presidential Democracy: The Case of Latin America, Baltimore: Johns Hopkins University Press, 1994, S. 3-87.

Die Delegation ist ein übliches, aber umstrittenes Instrument jeder modernen Verwaltungspraxis, wodurch das Parlament bzw. der Kongress auf die Regierung oder direkt auf die Verwaltung gesetzgeberische Gewalt überträgt. Die ausführenden Organe sind damit in der Lage, allgemeine Rechtsnormen über diejenigen Bereiche zu erlassen, die im Prinzip einer Regelung durch das Parlament vorbehalten bleiben sollen. Über die Delegation gibt es eine umfangreiche Literatur. Einen kompletten Überblick gibt Gallego Anabitarte, der sie allerdings befürwortet: Gallego Anabitarte, Ley y reglamento en el derecho público occidental, Madrid: Instituto de Estudios Administrativos, 1971. Eine klassische Kritik im Sinne Lockes entwickelt Carleton Kemp Allen, Law and orders: an inquiry into the nature and scope of delegated legislation and executive powers in England, London: Stevens \& Sons, 1945. Für Argentinien s. Alberto Bianchi, La delegación legislativa. Teoría de los reglamentos delegados en la Administración Pública, Buenos Aires: Ábaco, 1990. Für Brasilien s. Carlos Roberto de Siqueira Castro, O Congresso e as Dele- 
seine Rolle als Verkörperung eines „Gemeinwillens“, sollte damit endgültig abgesichert werden. Unabsichtlich verursachte jedoch die Verfassungsreform auf diese Weise eine schwere „technische“ Krise des Parlaments, deren Lösung noch nicht abzusehen ist.

Seit Locke wird die Delegation als ernste Gefahr für die Macht des Parlaments und für seine Rolle als Volksvertretung beschworen. $\mathrm{Zu}$ einem Verbot ist es allerdings bisher in keinem Land mit Ausnahme Argentiniens gekommen, da die Funktionsfähigkeit der öffentlichen Verwaltung teilweise von den legislativen Befugnissen abhängt, die ihr das Parlament überträgt. Kritiker der Delegation empfahlen zwar immer wieder eine strenge Kontrolle und die größtmögliche Beschränkung dieser Praxis, nicht jedoch ihre direkte Untersagung (die in Argentinien wohlgemerkt einige Ausnahmen zulässt). Mit einer so drastischen Entscheidung versuchte die verfassungsgebende Versammlung, wie schon oben angedeutet, einen tiefgreifenden Wandel im Verhältnis von Exekutive und Legislative herbeizuführen. Die Exekutive und die öffentliche Verwaltung (die üblicherweise in Lateinamerika völlig der Exekutive unterstellt ist) sollten wieder in der Hauptsache zu ausführenden Organen werden, indem der Kongress die Rolle des Gesetzgebers im eigentlichen Sinne des Wortes übernimmt.

Anlass für den Versuch, die Beziehungen zwischen den zwei Staatsgewalten auf diese Weise zu transformieren, war nicht nur die o.g. Kontroverse über den Präsidentialismus sondern auch die spezifische Tatsache, dass der argentinische Kongress in den vergangenen fünfzig Jahren durch etliche Gesetze eine enorme Zahl an Kompetenzen, in vielen Fällen sehr weitreichenden Umfangs, auf die Exekutive übertragen hatte. Dies ging teilweise so weit, dass die Exekutive zusammen mit den verschiedenen Verwaltungsbehörden als ,delegierte" Legislativgewalt für praktisch alle Sachgebiete wirken konnte.

Nun wäre es ohne Frage müßig gewesen, die Delegation für die Zukunft zu verbieten, aber die weiterhin geltenden Gesetze, die umfassende Delegationen an die Exekutive enthalten, bestehen zu lassen. Die reformierte Verfassung verbietet daher nicht nur die Übertragung rechtsetzender Kompetenzen an die Regierung. ${ }^{4}$ Sie entzieht darüber hinaus ausdrücklich

gações Legislativas: limites do poder normativo do executivo, Rio de Janeiro: Forense, 1986. Für Deutschland und die USA vgl. zwei komparative Untersuchungen: Thomas Fleiner, Die Delegation als Problem der Verfassungs- und Verwaltungsrechts. Ein rechtsvergleichender Beitrag zur Rechtsprechung auf dem Gebiet der Gewaltentrennung, Fribourg: Universitätsverlag, 1972, und Uwe Kischel, Delegation of Legislative Power to Agencies: A Comparative Analysis of United States and German Law, Administrative Law Review (Chicago), Bd. 46 (Frühling 1994), S. 213256.

Die Ausnahmen sind zwei: determinierte Verwaltungsangelegenheiten und Notstand. „Determinierte Verwaltungsangelegenheiten“ (materias determinadas de administración) ist ein deutlich auslegungsbedürftiger Ausdruck. Bisher war es jedoch nicht nötig, eine Interpretation verbindlich festzulegen, weil aus Gründen, die unten erörtert werden, die alte Delegationspraxis noch größtenteils erhalten bleibt. 
allen Gesetze, die Delegationen enthalten, und allen anderen Normen (Dekrete, Rechtsverordnungen), die aufgrund von Delegationen erlassen worden sind, innerhalb von fünf Jahren ihre Gültigkeit, sollten sie nicht durch ein neues Gesetz ratifiziert werden.

Dem Kongress wurde auf diese Weise eine vergleichsweise kurze Frist gesetzt zur Revidierung seiner Delegationspolitik in den letzten fünfzig Jahren. Dementsprechend hatte der Kongress zunächst die Aufgabe zu bewältigen, alle Dekrete und Rechtsverordnungen zu erfassen, die aufgrund seiner Ermächtigungen von der Exekutive und von der Verwaltung in der Vergangenheit erlassen worden waren. Denn die Situation war damals äußerst konfus: Niemand wusste genau, welche Befugnisse man eigentlich auf die Exekutive und auf die Verwaltungsbehörden übertragen hatte. Eine neuere Untersuchung kalkuliert, dass sich zum Zeitpunkt der Verfassungsreform von 1994 die Zahl der geltenden Gesetze auf etwa 2500 belief, von denen 1900 Delegationen an den Präsidenten, die Minister oder die Leiter von Regierungsbehörden enthielten. ${ }^{5}$

Die Anzahl von Delegationen oder von Gesetzen, die Delegationen enthalten, muss jedoch nur als eine Mutmaßung betrachtet werden, denn genauere Kenntnisse über die Rechtslage gab es damals noch nicht. Umfang und sogar Inhalt der Delegationen waren dem Kongress nur fragmentarisch bekannt.

Der Kongress musste außerdem darauf vorbereitet sein, die aufgehobenen Rechtsnormen unmittelbar ersetzen oder ratifizieren zu können, um eine Periode der Rechtsunsicherheit oder sogar eines juristischen Chaos zu vermeiden, das möglicherweise durch das plötzliche Verschwinden von bisher geltendem Recht entstehen könnte. Konkret bedeutete dies: Das gesamte argentinische Recht musste systematisch gesammelt und analysiert werden. Bis zum Ablauf der Fünfjahresfrist, also bis zum 25. August 1999, wurde allerdings kaum etwas von diesen Aufgaben erledigt. Der Kongress war daher überhaupt nicht fähig, zu entscheiden, welche Normen von der Verfassungsvorgabe ausgenommen waren und welche unverzüglich aufgehoben werden mussten. Allein faktisch war der Kongress technisch außerstande, die von ihm an die Regierung und Verwaltung übertragenen Kompetenzen wieder selber zu übernehmen.

Nun hatte der Kongress eigentlich schon im Mai 1998 ein Gesetz verabschiedet, das die Erarbeitung einer systematischen, elektronisch erfassten Datenbank in Auftrag gab. Diese sollte die alle geltenden (allgemeinen) Gesetze, alle allgemeinen Dekrete, die sich mit 
einem Gesetz durch Delegation oder Reglementierung verbinden, ${ }^{6}$ sowie alle entsprechend von der öffentlichen Verwaltung erlassenen allgemeinen Verordnungen zusammenstellen. Durch eine schnelle Umsetzung dieses Gesetzes hätte man das nötige Instrument schaffen können, um dem Kongress die Wiederaufnahme seiner gesetzgeberischen Gewalt zu ermöglichen.

Aber erst im Juli 1999 nimmt unter dem Namen Digesto Jurídico Argentino eine Gruppe von dreißig Juristen, darunter der Autor des vorliegenden Artikels, die systematische Aufarbeitung des Gesamtkorpus des argentinischen Rechts von seinen Anfängen im Jahre 1852 bis heute auf. Der Digesto Jurídico Argentino soll die einzige Rechtsquelle für geltendes Bundesrecht in Argentinien werden. Es handelt sich um ein bislang für Lateinamerika einmaliges Vorhaben, das in drei Jahren durchgeführt werden soll. In diesem elektronischen Corpus Juris sollen alle Delegationen der Vergangenheit sichtbar und zugreifbar werden, zusammen mit den daraus entstandenen Dekreten und Verordnungen. Damit könnte der Kongress nicht nur jede einzelne Delegation neu überdenken und eventuell aufheben, sondern auch das daraus entstehende Recht (Dekrete und Verordnungen) ersetzen. Denn dies ist die größte Schwierigkeit bei einem solchen Vorgehen: Ein Gesetz, das nach neueren Überlegungen des Kongresses übertriebene Delegationen enthält, könnte nicht einfach außer Kraft gesetzt (derogiert) werden, ohne die von diesem Gesetz abhängenden Dekrete und Verordnungen sofort zu ersetzen, zu reformieren oder zu ratifizieren. Solche Normen verlieren sonst urplötzlich ihren Status als geltendes Recht, und so verschwindet möglicherweise die Rechtsgrundlage gewisser Bereiche oder Institutionen der Gesellschaft. Deswegen wäre es völlig vermessen, ein Gesetz abzuschaffen, das Delegationen enthält, ohne die auf Grund dieser Delegation erlassenen Normen sehr genau zu erfassen. Mit anderen Worten: Die ganze Kette der Delegation muss zur Kenntnis genommen werden, bevor man den Anfang dieser Kette kappt.

Im August 1999 blieb dem Kongress demnach nichts anderes übrig, als alle in der Vergangenheit erlassenen Delegationen in ihrer Gesamtheit für drei Jahre zu ratifizieren. Diese Ratifikation war gewiss problematisch (das Gebot der Verfassung spricht deutlich gegen so eine allgemeine und blinde Ratifizierung), der Kongress hatte jedoch keine andere (realistische) Wahl. In der Zwischenzeit sollte die Analyse und „Konsolidierung“ (wie es technisch heißt) des argentinischen Rechts in Digesto Jurídico Argentino zu Ende gebracht werden.

Die Kompetenz der Exekutive, Gesetze zu „,reglementieren“ (facultad reglamentaria) ist ein spezifisches Merkmal des öffentlichen Rechts in Lateinamerika, von dem noch die Rede sein wird. Diese Reglementierungskompetenz funktioniert in der Praxis wie eine Delegation, obwohl das Thema etwas umstritten ist . Einige argentinische Autoren benutzen hier den etwas barocken Ausdruck von ,uneigentlicher Delegation“, s. Eugenio Luis Palazzo, Caducidad de las delegaciones legislativas. Presentación del tema - Una interpretación restrictiva, El Derecho. Diario de Jurisprudencia y Doctrina (Buenos Aires), Nr. 9768 - Jahrgang XXXVII (7.6.1999) Extra Reihe Verfassungsrecht, S. 1-4. 
Mit dem Auftrag für die Fertigstellung des Digesto Jurídico Argentino hatte der argentinische Kongress zwar die politische Bedeutung der Krise eindeutig erkannt, nicht jedoch ihr technisches Ausmaß. Die Gruppe von Juristen, die das Gesamtkorpus des argentinischen Rechts aufarbeiten soll, besteht aus lediglich dreißig Experten. ${ }^{7}$ Es ist sicher, dass diese Gruppe von Juristen aus dem Gesamtkorpus von etwa 2500 Gesetzen bestimmen kann, welche Gesetze Delegationen enthalten. Darüber hinaus wird es ihr wahrscheinlich auch gelingen, die Verbindung aller Gesetze zu ihren jeweiligen Reglementierungsdekreten herzustellen. Es ist jedoch meiner Meinung nach völlig auszuschließen, dass ein so kleines Team alle Normen (Dekrete und insbesondere die zahllosen Rechtsverordnungen) analysieren und konsolidieren kann, die aufgrund dieser Delegationen erlassen wurden. Will das Parlament seine legislative Rolle in vollem Umfang wiederaufnehmen, bedarf es jedoch dieser Informationen.

Um sich eine genauere Vorstellung von dem hier nötigen Arbeitsumfang zu machen, reicht ein Blick auf die parlamentarischen Ausschüsse in England (select committees), die zusammen mit der Informationsbeschaffung über die Verwaltungsaktivitäten auch die Untersuchung der delegierten Normen durchführen. Dort beschäftigten sich in der Sitzungsperiode 1992-93 ca. 300 Parlamentsmitglieder (staff nicht mitgerechnet) mit der Politik und mit den Normen, die allein während dieses Zeitraums aufgrund legislativer Ermächtigungen von den Verwaltungsstellen erlassen wurden. Dabei wurden 129 Berichte mit einem Gesamtvolumen von 16.456 Seiten erstellt. ${ }^{8}$ Und dennoch halten viele Fachleute das britische System für unzureichend, eine Meinung, die 1996 auch vom Procedure Committee vertreten wurde. ${ }^{9}$

Die argentinische Parlamentskrise macht schließlich folgenden, sehr wesentlichen Punkt deutlich: Die Kontrolle der delegierten Gesetzgebung, d.h. der Normsetzungsbefugnisse der öffentlichen Verwaltung, stellt die mühseligste Aufgabe eines modernen Parlaments und gleichzeitig wahrscheinlich auch eine seiner wichtigsten Funktionen dar. Mit dieser Funktion kann man nicht eine kleine außerparlamentarische Gruppe von Juristen betrauen; sie bedarf vielmehr mindestens der Schaffung eines leistungsfähigen Systems von nach Sachthemen und Verwaltungsbereichen ausdifferenzierten Ausschüssen. Nichtsdestotrotz verkörpert das Projekt des Digesto Jurídico Argentino eine bedeutsame Lernerfahrung hin-

7 Diese Aussage basiert nicht nur auf persönlicher Beobachtung des Autors. Das Reglementierungsdekret 1050/98 zum Gesetz 24.967 „Digesto Jurídico Argentino“ schreibt in der Tat die Berufung einer Kommission von nur 26 Experten zur Erarbeitung dieses Corpus Juris vor. Als "Controller" der Gruppe von Rechtsanalysten wurde Dr. Enrique Zeitler (ein Jurist ersten Ranges) ernannt.

Philip Giddings, Select Committees and Parliamentary Scrutiny: Plus Ça Change, Parliamentary Affairs (Oxford), Bd. 47, Nr. 4 (Okt. 1994), S. 669-686, hier 681. 
sichtlich der technischen Aspekte eines parlamentarischen Ausschusssystems, das die normsetzenden Aktivitäten der Regierung und der Verwaltung erfassen und kontrollieren kann.

\section{Machtressourcen der lateinamerikanischen Parlamente}

Der friedliche Regierungswechsel Ende 1999 in Argentinien scheint auf jeden Fall erneut zu bestätigen, dass sich eine präsidiale Demokratie in Lateinamerika konsolidieren kann. Seit der (Re-)Demokratisierung 1983 wurde der argentinische Präsident zum vierten Mal in freien und fairen Wahlen bestimmt.

Argentinien erweist sich somit als weiteres Gegenbeispiel - neben etwa Brasilien und Chile - zu der oben erwähnten These des amerikanischen Politologen Juan Linz. Nach Meinung von Linz muss der lateinamerikanische Präsidentialismus als Ursache für einen Mangel an politischer Stabilität der Demokratien in dieser Region betrachtet werden. In der ersten Hälfte der neunziger Jahre hat die Verbreitung von Linz' These maßgeblich zu einer skeptischen oder sogar pessimistischen Haltung gegenüber der möglichen Konsolidierung der lateinamerikanischen Demokratien beigetragen. Im Sinne von Linz wurden zwei politische Phänomene der neunziger Jahre als Zeichen für eine Rückkehr zu autoritären Traditionen interpretiert: Erstens die Tendenz der Präsidenten (besonders in Argentinien und Brasilien), mittels Notstandsdekreten ihre politischen Strategien durchzusetzen und zweitens die scheinbare Passivität des Parlaments gegenüber diesem Regierungsstil der Exekutive.

Nun hat die politische Realität Linz' These eingeholt: Argentinien und eine Reihe von lateinamerikanischen Demokratien wurden nicht von der politischen Instabilität erfasst, die nach dieser These zu erwarten gewesen wäre; sie haben vielmehr mit relativem Erfolg schwierige Zeiten meistern können. Es stellt sich somit die Frage, ob im präsidialen Charakter der lateinamerikanischen Demokratien nicht gerade das Geheimnis für ihr Überleben liegt. Es ist in der Tat zu bezweifeln, ob der Kampf gegen die Hyperinflation der 80er und 90er Jahre, der das Interesse vieler sozialer Gruppen berührte, im Rahmen eines parlamentarischen Systems Erfolg hätte haben können. ${ }^{10}$ Eine Periode des sogenannten „Hyperpräsidentialismus" erscheint im Nachhinein betrachtet auf jeden Fall weniger schädlich für eine Demokratie als die Alternative, die sehr wahrscheinlich in einer andauernden institutionel- 
len Krise bestanden hätte. ${ }^{11}$ Schließlich sind einige lateinamerikanische Länder, die mit besonderem Erfolg ihre Wirtschaft während der 90er Jahre modernisieren konnten, jene mit ausgeprägten Präsidialsystemen. ${ }^{12}$ Dies gilt für Brasilien und Chile noch mehr als für Argentinien, dessen wirtschaftliche Lage seit vier Jahren durch eine andauernde Rezession beeinträchtigt wird.

Die Stabilität der präsidialen Demokratie sollte deshalb nach Meinung verschiedener Autoren als ein ,zentrales empirisches Fundament“ in der Diskussion der politischen Institutionen Lateinamerikas anerkannt werden. ${ }^{13}$ Darüber hinaus versucht eine neuere Studie von Krumwiede und Nolte ${ }^{14}$ zu zeigen, dass die Vorstellung eines allmächtigen Präsidenten in Argentinien und anderen lateinamerikanischen Ländern, der mit Dekreten an ohnmächtigen Parlamenten vorbei regiert, als ein Klischee betrachtet werden muss. Die Vorstellung von präsidialer Allmacht und die These der Instabilität des Präsidentialismus sind in der Tat eng verbunden und haben sich in der Vergangenheit gegenseitig verstärkt.

Die Parlamente in Lateinamerika sind nicht ohnmächtig. Um diese Frage angemessen zu diskutieren, muss man nach Meinung von Krumwiede und Nolte als Erstes zwischen verschiedenen Formen der Machtausübung unterscheiden. Denn eines ist sicherlich zu erkennen: die sogenannte „Gestaltungsmacht“ der lateinamerikanischen Parlamente ist gering. Mit Gestaltungsmacht des Parlaments ist seine Fähigkeit gemeint, auf die Gestaltung der Politik - insbesondere im Bereich der Gesetzgebung - wirksam Einfluss zu nehmen. Für die präsidialen Regierungssysteme Lateinamerikas ist es in diesem Sinne typisch, dass nicht das Parlament, sondern die Regierung die überwiegende Mehrheit der Gesetze - vor allem der wichtigen - einbringt. ${ }^{15}$

Nun haben die lateinamerikanischen Parlamente bisher keine Kompetenz oder Neigung gezeigt, alternativ zu der Regierung eine eigene politische Agenda zu gestalten. Jedoch ist ihre Fähigkeit, die politische Strategie des Präsidenten zu beeinträchtigen, nicht zu unterschätzen. Mit parlamentarischer Verhinderungsmacht ist also die Fähigkeit gemeint,

George Philip, The New Populism, Presidentialism and Market-Orientated Reform in Spanish South America, Government and Opposition (London). Bd. 33 Nr. 1 (Wint. 1998), S. 81-97, hier S. 82.

George Philip, The Dilemmas of Good Governance: A Latin American Perspective, Government and Opposition (London), Bd. 34, Nr. 2 (Frühling 1999), S. 226-242.

Dieter Nohlen, Presidencialismo versus parlamentarismo: dos enfoques contrapuestos, in: Dieter Nohlen / Mario Fernández (Hrsg.), El presidencialismo renovado. Instituciones y cambio político en América Latina, Caracas: Nueva Sociedad, 1998, S. 15-26.

Heinrich Krumwiede / Detlef Nolte, Die Rolle der Parlamente in den Präsidialdemokratien Lateinamerikas. Beiträge zur Lateinamerika-Forschung, Bd. 4, Hamburg: Institut für Iberoamerika Kunde, 2000. 
bestimmte politische Maßnahmen zu verhindern, indem die von der Regierung eingebrachten Gesetzesentwürfe nicht angenommen werden. Diese Verhinderungsmacht ist ein wichtiger politischer Faktor: Sie ermöglicht ein notwendiges Kontrollminimum und sie setzt der Macht des Präsidenten deutliche Grenzen. ${ }^{16}$

Jede Machtmessung zeigt, wie Krumwiede und Nolte bemerken, methodische Schwierigkeiten. Denn von erheblicher Bedeutung ist hier gerade der Bereich der „Nicht-Entscheidungen“. Manchmal wird nämlich die Verhinderungsmacht des Parlaments so ausgeübt, dass der Präsident eine bestimmte politische Entscheidung nicht trifft, weil er den Widerstand des Parlaments antizipiert. Die parlamentarische Verhinderungsmacht ist Bestandteil der politischen Realität, sie kann jedoch in diesem Fall nicht als empirisches Faktum konstatiert werden. ${ }^{17}$

Trotz dieser interessanten Feststellung von Krumwiede und Nolte kann m.E. eine relativ positive empirische Unterstützung für ihre These über die (beträchtliche) Verhinderungsmacht der lateinamerikanischen Parlamente ermittelt werden. Dies zeigt sich eben an der Anzahl oder an dem Prozentsatz präsidialer Gesetzesinitiativen, die vom Parlament nicht angenommen werden. In Argentinien wurden beispielsweise von 1983 bis 1997 ungefähr $60 \%$ der vom Präsidenten eingebrachten Gesetzesentwürfe vom Parlament verabschiedet. ${ }^{18}$ Entweder abgelehnt oder nicht angenommen wurden also $40 \%$ der Gesetzesinitiativen des Präsidenten, eine Zahl, die meiner Meinung nach als eine empirische Messung von Verhinderungsmacht bewertet werden kann. ${ }^{19} \mathrm{Zu}$ diesem Prozentsatz muss gewiss noch die Art von Verhinderung hinzugefügt werden, die sich nicht in einem abgelehnten Gesetzentwurf sondern in der Entscheidung des Präsidenten manifestiert, den Entwurf wegen des erwarteten Widerstands des Parlaments nicht einzubringen (die von Krumwiede und Nolte

16

17

18

19

Krumwiede / Nolte, op.cit. (Fn. 14), S. 104.

Krumwiede / Nolte, op.cit. (Fn. 14), S. 108.

N. Guillermo Molinelli et al., Congreso, presidencia y justicia en Argentina, Buenos Aires: Temas, 1999, S. 434

Verschiedene Studien beschreiben einzelne Fälle von parlamentarischer Blockierung der präsidialen Politik und die daraus folgenden Verhandlungen, die einen unilateralen Führungsstil vom Präsidenten unmöglich gemacht haben. Für Argentinien gibt Llanos einen Überblick von mehreren Fallstudien aus den letzten zehn Jahren: Mariana Llanos, Über Gesetze und Dekrete: Eine Neuinterpretation der Beziehungen zwischen Präsident und Kongress im Argentinien der 90er Jahre, in: Klaus Bodemer / Andrea Pagni / Peter Waldmann (Hrsg.), Argentinien heute, Frankfurt am Main: Vervuert, 2001 (im Druck). Castro Santos beschreibt ihrerseits die Exekutive-Legislative Beziehungen in Brasilien als ein „Muster von konfliktiver Interaktion“ und diskutiert auch verschiedene Konfliktfälle zwischen Exekutive und Legislative in den neunziger Jahren, wo die Legislative politische Alleingänge des Präsidenten effektiv hindern konnte: Maria Helena de Castro Santos, Governabilidade, Governança e Democracia: Criação de Capacidade Governativa e Relações Executivo-Legislativo no Brasil Pós-Constituinte, Dados. Revista de Ciencias Sociais (Rio de Janeiro), Bd. 40, Nr. 3 (1997), S. 335-376. 
erläuterte „Nicht-Entscheidung“). Es ist daher sinnvoll anzunehmen, dass die vom argentinischen Parlament verhinderten präsidialen Gesetzinitiativen mehr als $40 \%$ darstellen.

Die Zahl der vom Kongress nicht akzeptierten Gesetzesinitiativen des Präsidenten eröffnet eine interessante vergleichende Perspektive. Molinelli et al. kontrastieren sie mit den Werten für die parlamentarischen Demokratien Europas, wo die sogenannte 90\%-Regel besteht, d.h., dass ungefähr $90 \%$ der von der Exekutive eingebrachten Gesetzentwürfe vom Parlament angenommen werden. ${ }^{20}$ Dieser Vergleich kann selbstverständlich keine uneingeschränkte Gültigkeit beanspruchen, wird doch die Exekutive in einer parlamentarischen Demokratie in der Regel (sieht man von Minderheitsregierungen ab) von der stärksten Fraktion im Parlament gestellt, so dass hier nicht von einem in der Verfassung programmierten Konflikt zwischen beiden Gewalten gesprochen werden kann, wie dies bei präsidialen Systemen der Fall ist. Dennoch muss betont werden, dass das Parlament auch in den europäischen Demokratien seinen Anspruch auf eine (unabhängige) Kontrolle der Regierung nicht einfach aufgeben kann (oder darf). Ein wichtiges Kontrollminimum wird auf jeden Fall gewährleistet, so dass der Vergleich mit den präsidialen Demokratien Lateinamerikas einleuchtet, zumal von einigen Autoren die Existenz eines Kontrollminimums für gewisse lateinamerikanische Länder bestritten wird.

So behaupten beispielsweise Figueiredo und Limongi, dass der brasilianische Kongress die politischen Handlungen des Präsidenten nicht nur nicht verhindert, sondern im Gegenteil eher versucht, dem Präsidenten mögliche Hindernisse aus dem Weg zu räumen. ${ }^{21}$ Das von den Autoren dargestellte Bild des Kongresses erweckt den Eindruck, dass dieser dem Präsident völlig unterlegen ist. Trotz der emphatischen Argumente von Figueiredo und Limongi lassen sich jedoch einige Fakten, die in ihrer Studie von 1996 erörtert werden, auch so interpretieren, dass die theoretischen Schlussfolgerungen der Autoren nicht mehr empirisch abgesichert erscheinen. Während des Zeitraums 1989-1994 hat der brasilianische Kongress auf jeden Fall 51,7\% der vom Präsidenten eingebrachten Gesetzinitiativen (zu gewöhnlichen Gesetzen) nicht angenommen. ${ }^{22}$

Die unterschiedlichen Sichtweisen verschiedener Autoren sind bemerkenswert. Während für Molinelli et al. 40\% von verhinderten Gesetzesinitiativen des Präsidenten einen „,kom-

Argelina Cheibub Figueiredo / Fernando Limongi, Executivo e Legislativo na nova ordem constitucional, Rio de Janeiro: Editora FGV, 1999, S. 42.

22

Figueiredo / Limongi, op.cit. (Fn. 21), S. 76. Bei den gewöhnlichen Gesetzen (leis ordinárias) werden die Bundeshaushaltsgesetze nicht berücksichtigt, da das Initiativrecht exklusiv beim Präsidenten liegt und über sie beide Kammer in Plenarsitzungen debattieren und abstimmen müssen. Die Notstandsdekrete (medidas provisórias) bleiben auch ausgeschlossen. 
parativ hohen Wert" darstellen, ${ }^{23}$ so dass sich der argentinische Kongress als stark widerstandsfähig erweist, beweist nach Meinung von Figueiredo und Limongi ein Anteil von 51,7\% verhinderten Gesetzinitiativen des brasilianischen Präsidenten das genaue Gegenteil: der Kongress ist dem Präsidenten ausgeliefert. Die Sichtweise von Molinelli et al. scheint nach Ansicht des Verfassers wesentlich plausibler: ein Anteil von 40\% von abgelehnten Initiativen lässt ohne Zweifel auf eine beachtliche Verhinderungsmacht schließen.

Angaben über den chilenischen Kongress bestätigen ebenfalls die Existenz einer beachtlichen Verhinderungsmacht des Parlaments: Während des Zeitraumes 1990-1993 wurden 36,6\% der vom Präsidenten eingebrachten Gesetzentwürfe nicht vom Kongress verabschiedet. ${ }^{24}$ Die Ergebnisse für die drei Parlamente werden in folgender Tabelle verglichen.

Tabelle 1: Verhinderungsmacht der Parlamente in Brasilien, Argentinien und Chile

\begin{tabular}{|l|c|c|c|}
\hline & $\begin{array}{c}\text { Brasilien } \\
(1989-1994)\end{array}$ & $\begin{array}{c}\text { Argentinien } \\
(1983-1997)\end{array}$ & $\begin{array}{c}\text { Chile } \\
(1990-1993)\end{array}$ \\
\hline $\begin{array}{l}\text { Verhinderungsmacht } \\
\text { (Prozentsatz der präsidialen }\end{array}$ & $51,7 \%$ & $40 \%$ & $36,6 \%$ \\
$\begin{array}{l}\text { Gesetzinitiativen, die vom } \\
\text { Kongress nicht angenommen } \\
\text { wurden) }\end{array}$ & & & \\
\hline
\end{tabular}

Quelle: Molinelli et al., op.cit. (Fn. 19), S. 96 (Argentinien); Figueiredo / Limongi, op.cit. (Fn. 21), S. 76 (Brasilien); Siavelis, op.cit. (Fn. 24), S. 24 (Chile).

Sowohl diese Zahlen als auch die o.g. Fallstudien unterstützen die These, dass die Parlamente der lateinamerikanischen Präsidialdemokratien einen nicht $\mathrm{zu}$ unterschätzenden Machtfaktor darstellen. Wenn wir nun von dieser These ausgehen, eröffnet sich aber eine neue und tiefer gehende Problematik: warum können die lateinamerikanischen Parlamente ihre Macht nur auf eine solche „,negative“ Weise ausüben? Denn die Gleichsetzung von parlamentarischer Macht mit Verhinderungsmacht schadet gewiss dem Ansehen der lateinamerikanischen Parlamente. Es entsteht die Gefahr, dass Parlamente von vielen Bürgern als bloße Obstruktionsfaktoren wahrgenommen werden. Dies ist umso bedenklicher, als Umfragen des „Latinobarómetro“ seit Mitte der 90er Jahre die Tendenz in Lateinamerika zeigen, der Exekutive mehr Vertrauen entgegenzubringen als dem Parlament. Das Vertrauen in die Exekutive steigt, während das Vertrauen in die Legislative tendenziell sinkt. Auch der Justiz wird insgesamt in Lateinamerika mehr Vertrauen entgegengebracht als der

Peter M. Siavelis, The President and Congress in Post-Authoritarian Chile: Institutional Constraints to Democratic Consolidation, University Park (Pennsylvania): Penn State University Press, 2000, S. 24. 
Legislative. Eine starke Minderheit (von fast 30\%) der Befragten vertritt außerdem die Meinung, dass die Parlamente für die Demokratie entbehrlich sind. ${ }^{25}$

Die Frage nach den möglichen Ursachen für das Primat einer „obstruktiven“ Art der Machtausübung bei den lateinamerikanischen Parlamenten hat unter diesen Umständen eine wesentliche Bedeutung für die Forschung über die politischen Institutionen der Region. Nach dem folgenden „Exkurs“ über die Regierungskrise Argentiniens Dezember 2001 / Januar 2002, der ein konkretes Beispiel parlamentarischer Macht und gleichzeitig eine „Paradoxie“ der Delegation legislativer Gewalt darstellen soll, wird auf die Problemstellung des Primats einer Verhinderungsmacht eingegangen werden.

\section{Exkurs: Machtübernahme des Kongresses? Die Regierungskrise Argentiniens im Dezember 2001 / Januar 2002}

In der jüngsten Regierungskrise Argentiniens zeigten sich die Stärke und gleichzeitig auch die schwerwiegenden Defizite der parlamentarischen Macht in einer lateinamerikanischen Präsidialdemokratie.

Ausgangspunkt der Krise war der Rücktritt des Präsidenten de la Rúa am 20. Dezember, eine direkte Folge der seit Jahren andauernden Rezession und der hohen Auslandsverschuldung, die zur Zahlungsunfähigkeit des argentinischen Staates Mitte Dezember führten. ${ }^{26}$ Jede präsidiale Verfassung beinhaltet nun die Figur eines Vizepräsidenten, der die Präsidentschaft in solchen Fällen übernehmen soll. De la Rúas regierte jedoch ohne einen Vizepräsidenten, denn dieser war als Folge einer Konfrontation mit dem Oberen Haus des Parlaments (Senat) schon in vorigen Jahr zurückgetreten.

Nach dem Rücktritt de la Rúas wurde also die Präsidentschaft direkt vom Vorsitzenden des Senats übernommen, so wie es das argentinische Gesetz Nr. 20.972 zur „Führungslosigkeit" (Ley deAcefalía) vorsieht. Einige Tage danach ernannten beide Kammer des Kongresses in Plenarsitzung - auch nach Gesetzesvorschrift - einen Präsidenten, Adolfo Rodriguez Saá. Der neue Präsident sollte jedoch die Regierung für nur etwas mehr als drei Monate übernehmen, damit schnell neue Präsidenschaftswahlen ausgeschrieben werden könnten. Denn die grundlegende „Logik“ einer Präsidialverfassung besteht in der Tat darin, Präsident und Kongress durch getrennte Volkswahlen jeweils eine eigene demokratische Legitimation zu sichern. 
Nach einer Woche musste jedoch der Kongress erkennen, dass Präsident Saá dem Ernst der wirtschaftlichen und politischen Lage nicht einmal für zwei Monate gewachsen war. Und schlimmer noch: Einige politische Akteure, darunter auch der ehemalige Präsident Carlos Menem (1989-1998), plädierten dafür, dass der Präsident nicht wie vereinbart bis zum 3. März 2002 sondern bis zum Ende der ursprünglich vorgesehenen Amtsperiode von de la Rúa (2003) im Amt bleiben solle. Dies bedeutete jedoch eine Aberkennung der parlamentarischen Bedingungen für seine Nominierung und dem Präsidenten Saá wurde aus beiden Gründen jede politische Unterstützung entzogen. Er trat am 30. Dezember zurück.

Diesmal übernahm für zwei Tage der Vorsitzende der Abgeordnetenkammer die Präsidentschaft, bis beide Kammern in Plenarsitzung einen Präsidenten ernennen konnten. Hier wurde also nicht mehr nach dem Gesetz entschieden, denn dieses hätte eine erneute Designation des Senatsvorsitzenden vorgeschrieben, sondern nach den Machtverhältnissen im Kongress: der Vorsitzende der Abgeordnetenkammer, Eduardo Camaño, gehört zu der stärksten parlamentarischen Fraktion innerhalb der peronistischen Partei, die vom (damaligen) Senator Eduardo Duhalde geführt wird. Duhalde konnte während dieser zwei Tage eine große parlamentarische Koalition mit der Opposition (UCR und Frepaso, die Regierungsparteien vor dem Rücktritt de la Rúas) vereinbaren, und so wurde er mit 262 vonr 301 Stimmen am 1. Januar vom Kongress zum Präsidenten gewählt.

Nun wurde Präsident Duhalde von vornherein nicht für eine kurze Zeit berufen, so dass Präsidentschaftswahlen möglichst schnell stattfinden konnten. Er wurde - so wie Saá es für sich gewollt hätte - bis zum Ende der ursprünglich vorgesehenen Amtsperiode de la Rúas (Ende 2003) ernannt. Seine Legitimation gründet sich damit nicht auf einer Volkswahl, so wie es eine Präsidialverfassung vorsieht, es handelt sich hier vielmehr - und sei es nur für eine relativ kurze Zeit - um eine parlamentarische Regierung.

Inwiefern jedoch kann der Kongress diese Regierung politisch kontrollieren? Gleicht der Kongress nicht eher einer ,glorifizierten Wahlversammlung“, wie von einigen Volksvertretungen parlamentarischer Systeme behauptet wird? Solche Parlamente - so wird weiter eingewandt - sind nur für die Dauer eines Augenblicks mächtig, denn ist erst einmal das Staatsoberhaupt gewählt, dann müssen sie sich diesem völlig unterordnen.

Für diese Interpretation könnte die Tatsache sprechen, dass der Kongress am 6. Januar weitreichende Gesetzgebungskompetenzen an den Präsidenten übertragen hat. Durch das Gesetz 25.561 wurde zuerst der ,öffentliche Notstand“ erklärt (Art. 1). Der Notstand stellt eine der zwei Bedingungen dar, unter denen das Delegationsverbot der argentinischen Verfassung aufgehoben werden kann. Eine andere Ausnahme dieses Verbots betrifft die Delegation gesetzgeberischer Gewalt im Bereich ,determinierter Verwaltungsangelegenheiten“ (ein Begriff, der sich eher undeutlich auf Verwaltungsgesetze beziehen lässt). Nach Erklärung des öffentlichen Notstands ermächtigt also Art. 2 des Gesetzes den Präsidenten, 
„ein System zu etablieren, das den Wechselkurs zwischen Peso und Auslandswährungen bestimmen soll“ sowie „Wechselkursreglementierungen zu erlassen.“ Diese Materien stellt die argentinische Verfassung eigentlich unter Gesetzvorbehalt, doch aufgrund der Delegation werden sie nun vom Präsidenten direkt reglementiert.

Weitere Delegationen im Gesetz betreffen unmissverständlich Grundrechte. Art. 6 ermächtigt den Präsidenten, Schulden in Dollar oder anderen Auslandswährungen zum neuen Wechselkurssystem ,anzupassen“. Auf diese Weise können alle Verpflichtungen (private oder staatliche), die in Dollar vereinbart wurden, zwangsweise von der Exekutive in die argentinische Währung Peso umgetauscht werden. Private Bankkonten können weiterhin von der Exekutive ,restrukturiert“ werden. Das Eigentumsrecht über Schulden in Auslandswährung und Bankkonten wird mit anderen Worten teilweise aufgehoben.

Um die Gläubiger zu kompensieren, wird der Präsident weiterhin ermächtigt, öffentliche Anleihen zu emittieren und diese durch eine neue Steuer auf den Ölexport oder durch Auslandskredite zu garantieren. Schließlich wird der Präsident zur Bestimmung der Preise von „kritischen“ Waren und Dienstleistungen ermächtigt (Art. 13). Das Eigentumsrecht wird hiermit auch für Güter und Dienstleistungen teilweise aufgehoben.

Die vom Gesetz 25.561 delegierten Gesetzgebungskompetenzen sind ohne Frage weitreichend und gravierend. Und gewiss ist der Kongress nicht imstande, die technischen Einzelheiten bei der Anwendung dieses Gesetzes zu kontrollieren. Denn die Vorbedingung einer solchen Kontrolle sind die Ziele der Delegation, die im Gesetz als ein Programm vorgelegt werden. Das Programm des Gesetzes dient als Maßstab für den richtigen Gebrauch der delegierten Kompetenzen und ermöglicht somit eine technisch detaillierte parlamentarische Kontrolle der Exekutive. Das wurde in diesem Falle nicht getan. Eine Art Programm beinhaltet nur der Artikel 1 des Gesetzes 25.561 und hier werden z. B. als Ziele der Delegationen erklärt, dass damit die Exekutive „die Wirtschaft zu reaktivieren“ hat, das „Niveau der Beschäftigung und die Distribution von Gütern verbessern“ soll, ,den Akzent auf die Entwicklung der regionalen Wirtschaft“ zu setzen hat, die „Bedingungen für ein nachhaltiges Wirtschaftswachstum schaffen“ muss, usw. Zur Gestaltung der Politik ist der Beitrag des Kongresses mit diesen allgemeinen Grundsätzen und rhetorischen Deklarationen gleich null. In diesem angeblich programmatischen Artikel zeigt sich nur - und sehr deutlich - die fehlende Gestaltungsmacht des argentinischen Kongresses.

Es kann wohl gefragt werden, ob der Kongress mit solchen weitreichenden Delegationen seine eigene Machtposition als veto player, seine Verhinderungsmacht nicht nachhaltig beschädigt hat. Kündigen etwa diese umfassenden Vollmachten eine Flucht in einen „reinen“, ,,absoluten“ Präsidentialismus? Oder handelt es sich nur um Zugeständnisse im Rahmen der Krisenbewältigung? 
Die Antwort auf diese Fragen mag auf den ersten Blick paradox erscheinen: Der Kongress hat mit diesen Delegationen seine Machtposition als veto player gegenüber dem Präsidenten nicht im geringsten geschwächt. Denn die Delegation kann sehr wohl als ein Machtinstrument vom Parlament benutzt werden. Ein kurzer Rückblick auf die letzte Phase der Regierung de la Rúas bestätigt diese Annahme: Der Argentinische Kongress hat gegenüber der Exekutive die Delegation als Instrument seiner (Verhinderungs-) Macht sehr effektiv nutzen können. ${ }^{27}$ Denn an de la Rúa wurden im März 2001 sehr umfassende Gesetzgebungskompetenzen übertragen, die bei weitem diejenigen übertreffen, die Präsident Duhalde nun erhalten hat.

Die Regierung des Präsidenten Duhalde wurde oben als parlamentarisch definiert, weil der Präsident direkt vom Kongress und nicht durch Volkswahlen ernannt wurde. Dieser Machtzuwachs des Kongresses hat wohlgemerkt eine Vorgeschichte oder Übergangsperiode. Die letzte Phase der Präsidentschaft de la Rúas kann indessen als eine semi-parlamentarische (oder auch semi-präsidiale) Regierung bezeichnet werden. Diese Periode begann am 19. März 2001 mit der Ernennung von Domingo Cavallo zum Wirtschaftsminister. Auch wenn er das Amt des Kabinettchefs nicht innehatte, übernahm Cavallo dennoch die Rolle eines Kanzlers oder Regierungschefs und so wurde er als der „Superminister“ der Regierung bezeichnet. Die Gründe für seine Berufung waren unmissverständlich: Präsident de la Rúa wollte die Unterstützung der parlamentarischen Opposition, d.h. der peronistischen Partei, gewinnen und dieses Ziel wurde in der Tat erreicht Einen Konflikt mit seiner eigenen Partei, d.h. mit der Radikalen Bürgerunion (UCR), musste der Präsident dafür in Kauf nehmen. Denn Cavallo wurde als erfolgreicher Wirtschaftsminister des peronistischen Präsidenten Carlos Menem 1991-1996 berühmt und, obwohl kein Mitglied der Partei, hatte er seitdem ausgezeichnete Beziehungen zu den Spitzenfiguren unter den Peronisten. De la Rúa bildete, mit anderen Worten, eine neue parlamentarische Koalition.

Am 29. März 2001 verabschiedete der Kongress das Gesetz 25.414, das für Cavallo die Vorbedingung für die Annahme des Ministerpostens war. Hier einige Beispiele von den Gesetzgebungskompetenzen, die durch dieses Gesetz an die Exekutive übertragen werden: Im Art. 1 wird der Präsident dazu ermächtigt: a) Alle Regierungsbehörden entweder zu fusionieren oder zu spalten und ihre juristische Struktur zu ändern; b) Den Kapital- und Versicherungsmarkt zu deregulieren; c) Das Ministeriengesetz zu ändern; d) Verwaltungsgesetze (mit einigen bestimmten Ausnahmen) teilweise oder völlig aufzuheben; e) Steuern zu reduzieren und Steuerbegünstigungen zu schaffen oder aufzuheben; f) Sondersteuern zur Finanzierung von Infrastrukturprojekten einzuführen; g) Gesetze teilweise oder völlig aufzuheben (mit einigen bestimmten Ausnahmen), wenn solche Gesetze ,die Konkurrenzfähigkeit der Wirtschaft" beschädigen, usw. Die Ziele dieser Delegationen, das Programm des Gesetzes, wird im Gesetzestext nicht erwähnt. 
Präsident Duhalde hat Anfang Januar vom Kongress die Befugnis dafür erhalten, ein Wechselkurssystem zu etablieren sowie Schulden in Auslandswährungen und Bankkonten teilweise zu enteignen. Dazu kommt die Befugnis zur Einführung einer staatlichen Preisregulierung (bisher nicht angewandt). Obwohl diese Kompetenzen weiteichend sind, so sind sie jedoch relativ konkret und als Instrumente zur Bewältigung einer bestimmten Wirtschafts- und Finanzkrise konzipiert. Darüber hinaus können m. E. diese Befugnisse per se die Machtposition der Exekutive nicht stärken. Der Wirtschaftminister, oder besser gesagt „Superminister“ Cavallo bekam dagegen vom Kongress Befugnisse solcher Art, die erstens eine völlige Restrukturierung der öffentlichen Verwaltung und zweitens eine grundlegende Reform sowohl des Steuersystems als auch des ganzen Wirtschaftsrechts seitens der Exekutive möglich machten.

Solche umfassenden Befugnisse sind jedoch ein zweischneidiges Schwert, wie Cavallo und der de la Rúa später erkennen mussten. Werden sie einmal vom Kongress zurückgefordert, verliert die Regierung ihre Funktionsfähigkeit und ihr bloßes Überleben wird fraglich. Denn weitreichende Delegationen verwandeln teilweise die Natur einer präsidialen Regierung. Die Volkswahlen bilden nicht mehr die einzige Legitimationsbasis des Präsidenten. Seine Regierung wird von der parlamentarischen Mehrheit abhängig.

Im Jahre 1999 gewann de la Rúa die Präsidentschaftswahlen mit 48\% der Stimmen. Die massiven Demonstrationen gegen seine Regierung am 19. und am 20. Dezember 2001 stellen unzweifelhaft einen der Gründe für seinen Rücktritt dar. Nicht weniger entscheidend war jedoch ein buchstäblicher Aufstand des Parlaments in jenen Tagen. Nicht nur wurden die an die Exekutive übertragenen Gesetzgebungskompetenzen durch Derogation des Gesetzes 25.414 zurückgefordert. Das Parlament hat darüber hinaus am 20. Dezember eigenhändig die Verlängerung seiner Sitzungen beschlossen. Nach der Verfassung darf der Kongress Wiederbeginn und Schluss der Sitzungen jeweils am 1. März und am 30. November bestimmen. Eine Verlängerung der Sitzungen oder die Einberufung von Sondersitzungen liegen dagegen nur in der Zuständigkeit des Präsidenten (Art. 63 der argentinischen Verfassung). Die Regierung hatte schließlich nicht nur die Kompetenzen verloren, die eine nötige Bedingung ihrer Politik gewesen waren. Die Regierung stand nun auch einem Kongress gegenüber, der die faktische Unabhängigkeit seiner eigenen Macht erklärt hatte. Dem Präsidenten blieb indessen nichts anderes übrig, als nach Hause zu gehen.

Eine alternative Politik konnte das Parlament jedoch nicht anbieten. Die Ernennung eines Präsidenten für eine Woche zeigt hier in der Tat eine grosse Orientierungslosigkeit. Die Politik der teilweise parlamentarischen Regierung Cavallos wurde ab Oktober 2001 vom Parlament heftig kritisiert und schliesslich wurde die Regierung selbst gestürzt. Für eine Machtübernahme, die aus diesen Ereignissen resultieren musste, war der Kongress allerdings völlig unvorbereitet. Nach einer Woche war es deutlich geworden, dass Präsident Saá die nötige Improvistationskunst fehlte. Präsident Duhalde vermochte zumindest einen Plan 
vorzulegen, selbst wenn dieser sehr ungenau und fragmentarisch geblieben ist. Damit konnte er jedoch die üblichen Gesetzgebungsbefugnisse vom Kongress erhalten. Zur einer aktiven Gestaltung der Politik dieser Regierung, die von ihm selbst ja gebildet wurde, hat der Kongress jedoch nicht beigetragen. Im folgenden Punkt werden die möglichen Gründe für diese merkwürdige Einseitigkeit der parlamentarischen Macht untersucht.

\section{Konzeptionen der Parlamentsarbeit in Lateinamerika}

Zur Erklärung der „obstruktiven“ Eigenart der Parlamentsarbeit in Argentinien, Brasilien, Chile und anderen lateinamerikanischen Ländern muss als erstes gewiss die Tatsache erwähnt werden, dass die Exekutivgewalt als Institution während der häufigen Militärdiktaturen in Lateinamerika uneingeschränkt weiter funktioniert hat und ihre Verfügungsgewalt über Beamte und Experten größtenteils erhalten oder ausbauen konnte. ${ }^{28}$ Bei den parlamentarischen Beratungs- und Informationsdiensten gibt es dagegen während einer Diktatur einen deutlichen Kontinuitätsbruch, der nach der Demokratisierung erst überbrückt werden muss.

Nun stellt die Verhinderungsmacht im Vergleich zur Gestaltungsmacht keine größeren Ansprüche an Beratung und Information. Ohne diese Ressourcen musste sich das Parlament nach der Demokratisierung auf eine ,passive“ oder ,negative“ Rolle beschränken: Die Pläne des Präsidenten wurden eventuell verhindert oder vereitelt, die politische Agenda jedoch weiter von diesem bestimmt. Inzwischen hat sich mit den Jahren eine Art ,falsches Selbstverständnis“ der Parlamentarier entwickelt, die zu der Meinung neigen, dass ihrer Aufgabe durch das bloße Verhindern von unerwünschten präsidialen Initiativen Genüge getan wird. Auf diese Weise wird die parlamentarische Arbeit selbst nach Jahren nicht professionalisiert: Nicht nur effiziente Beratungs- und Informationsdienste scheinen teuer und überflüssig zu sein, sondern auch die Professionalisierung der Parlamentarier selbst wird nicht gefördert. ${ }^{29}$

Um nur ein Beispiel zu nennen: der Wirtschaftsminister Argentiniens unter dem ehemaligen Präsidenten Menem (1989-1998), Domingo Cavallo, dessen Konvertibilitätsplan noch bis Ende Dezember 2001 fortbestand, konnte während der letzten Diktatur wichtige technische Positionen innerhalb der exekutiven Gewalt übernehmen, bis zur Leitung der Zentralbank. Diese Erfahrung ist für seine spätere Rolle in der Demokratie sicherlich nicht belanglos gewesen. Unter dem Präsidenten de la Rúa (1998-2001) wurde Cavallo wieder zum Minister ernannt, was seine Rolle als „unparteilicher“ Experte bestätigte. Der katastrophale Misserfolg seiner Wirtschaftspolitik hat jedoch das Ansehen Cavallos dauerhaft beschädigt.

S. Mark P. Jones et al., Professional Politicians - Amateur Legislators: The Argentine Congress in the $20^{\text {th }}$ Century, Paper prepared for presentation at the Annual Conference of the International Society of New Institutional Economics (Manuskript), Tübingen, Deutschland, September 22-24, 2000 (Eine spanische Übersetzung dieses Textes kann von der Internet Adresse der „Fundación Gobierno y Sociedad“ heruntergeladen werden: http://www.fgys.org/docum/docum.htm). Hier 
Offensichtlich muss diese einseitige Rolle des Parlaments verändert werden. Jede Verbesserung in diesem Bereich muss jedoch von der Einsicht ausgehen, dass die Parlamentarier ihre Rolle als Kontrolleur und Gegengewicht der Exekutive wahrnehmen, weil sie sie wahrnehmen können und wahrnehmen wollen. Problematisch ist also eigentlich weniger der Mangel an politischem Ehrgeiz oder an politischen Machtmitteln der Parlamentarier, als vielmehr eine sehr eingeschränkte Konzeption der parlamentarischen Arbeit. Den technischen Hintergrund einer solchen Konzeption bilden nicht nur die vorher erwähnten Unterbrechungen und der Abbau von parlamentarischen Infrastrukturen während der Militärdiktatur, sondern auch die regelmäßige und häufige Anwendung einer für Lateinamerika charakteristischen präsidialen Befugnis, der bisher jedoch wenig Aufmerksamkeit in der Literatur gewidmet wurde: Der Kompetenz der Exekutive, Gesetze zu „reglementieren“ (Reglementierungskompetenz).

Dass der lateinamerikanische Präsidentialismus im wesentlichen von der Verfassung der USA inspiriert wurde, pflegt die übliche Erklärung für die Entstehung dieser Regierungsform zu sein. Es gibt jedoch einen entscheidenden Unterschied zwischen beiden Systemen, denn die Reglementierungskompetenz ist in den USA unbekannt. Diese Befugnis geht auf die spanische Verfassung von 1812 (Verfassung von Cádiz) zurück und findet sich in mehreren lateinamerikanischen Verfassungen wieder. Beispielhaft seien hier genannt: die Verfassung von Brasilien Art. 84, die Verfassung von Argentinien Art. 99, die Verfassung von Chile Art. 32, die Verfassung von Peru Art. 118, die Verfassung von Uruguay Art. 168. In der mexikanischen Verfassung (Art. 89) wird eine solche Reglementierungskompetenz der Exekutive nicht explizit gemacht, sie wurde jedoch so von der Rechtsprechung interpretiert. $^{30}$

Worin besteht nun die Reglementierungskompetenz? Mit diesem Terminus wird die Befugnis der Exekutivgewalt bezeichnet, nach Verabschiedung eines Gesetzes „Reglementierungsdekrete“ zu diesem spezifischem Gesetz zu erlassen. Im allgemeinen sind Reglementierungsdekrete sehr viel ausführlicher und präziser als das Gesetz selbst. Es wird von der Exekutive nämlich erwartet, dass sie sich all jener Details annimmt, die der Gesetzgeber nicht vorsehen konnte oder wollte. Darüber hinaus können die Reglementierungsdekrete das Gesetz interpretieren, sein Anwendungsgebiet erweitern oder einschränken.

Ausführende Verordnungen sind selbstverständlich nicht nur in Lateinamerika eine typische Ergänzung zu fast allen Gesetzen. Spezifisch an Lateinamerika ist die Tatsache, dass

werden Wahlsysteme in Argentinien als weitere mögliche Ursache für den Mangel an Professionalisierung der Abgeordneten untersucht.

S. David Pantoja Morán, El diseño constitucional del ejecutivo en México y sus problemas. Una revisión histórica, Foro Internacional. Revista trimestral publicada por El Colegio de México (Mexiko), Bd. XXXVIII, Nr. 2-3 (April-September 1998), S. 248-301, hier S. 272. 
die Exekutive überhaupt keine Extra-Befugnis oder Delegation vom Parlament braucht, um ausführende Rechtsverordnungen (oder besser gesagt Reglementierungsdekrete) zu einem Gesetz zu erlassen. Die Reglementierungskompetenz leitet sich aus der Verfassung ab und sie wird häufig und in vollem Umfang von der Exekutive ausgeübt und von der Legislative erwartet. Auf diese Weise wird in Lateinamerika die Reglementierungskompetenz als eine praktische Notwendigkeit angesehen und diese Betrachtungsweise hat zu einer sehr toleranten Jurisprudenz bezüglich ihrer richterlichen Kontrolle geführt.

Im Rahmen der regelmäßigen Ausübung der Reglementierungskompetenz wurde die Modernisierung der lateinamerikanischen Staaten im 20. Jahrhundert schließlich so durchgeführt, dass sowohl die Fachkompetenzen bei der Normsetzung als auch die Kontrolle der Regierungsagenturen von der Exekutive monopolisiert wurden. Es ist daher überhaupt nicht verwunderlich, dass das Parlament mit der zunehmenden Komplexität der Normsetzung nicht zurecht kommt. Es verfügt nicht über die Informationen und das Sachpersonal, die - fast ausschließlich - der Exekutive zur Verfügung stehen.

Die Reglementierungskompetenz hat auf diese Weise den schon vorher erwähnten funktionalen Effekt: Eine geringe Fähigkeit des Parlaments, eigene Gesetzesentwürfe zu entwickeln oder die vom Präsidenten eingebrachten Entwürfe seinen Vorstellungen gemäß umzugestalten. Kurz gesagt, sie trägt zu der geringen Gestaltungsmacht der Parlamente bei.

In der Tat bestehen die Gesetze (oder die Reformvorschläge zu den Gesetzentwürfen der Regierung), die das Parlament in der Lage ist einzubringen, lediglich aus allgemeinen Grundsätzen oder sogar lediglich rhetorischen Deklarationen. Aufgrund der Reglementierungsbefugnis gehen die Parlamentarier nämlich davon aus, dass sich die Exekutive um die Details der zu regelnden Materie kümmern wird. Gesetze, die dieser Tendenz nicht entsprechen und ein größeres Maß an Genauigkeit aufweisen, werden deshalb oft von den Parlamentariern selbst als zu ,reglementaristisch“ kritisiert und abgewertet.

Da das Parlament sich lediglich darauf beschränkt, allgemeine Rechtsgrundsätze aufzustellen, reichen ihm grobe Informationen über das im Gesetz zu Regelnde aus. Es besteht von daher keine Notwendigkeit, fachliche Kenntnisse oder empirische Informationen über die Regierungsaufgaben zu erwerben bzw. zu sammeln. Dies hat wiederum zur Folge, dass der Gesetzgeber auf Expertenteams verzichten kann, oder anders gesagt: Die legislativen Aufgaben können von Amateurpolitikern effizient genug wahrgenommen werden. ${ }^{31}$ Die eigent-

Den Unterschied zwischen Amateur-Abgeordneten und Berufspolitikern analysieren auch Jones et al., op.cit. (Fn. 27). Diese Untersuchung bezieht sich jedoch vorwiegend auf die Wahl der Abgeordneten. Eine empirisch-vergleichende Studie über die Systeme der Parlamentsberatung führen Krumwiede / Nolte, op.cit. (Fn. 14), S. 158 ff. durch. Diese Autoren betonen auch das Phänomen, dass ein Parlament, das sich auf die Verhinderung beschränkt, weder Berufspolitiker noch Informations- und Beratungsstrukturen benötigt (a.a.O., S. 104). 
lich fachliche Kapazität zur Normsetzung konzentriert sich somit in der Bürokratie der öffentlichen Verwaltung, die unmittelbar von der Exekutivgewalt abhängt. Die einzigen Gesetzesvorschläge, die ein höheres Maß an Präzision aufweisen, sind von daher jene, die von der Regierung erarbeitet werden (und die später dann auch noch von ihr reglementiert werden können). Dem Gesetzgeber kommt lediglich die Aufgabe zu, diese Gesetze zu akzeptieren oder ihnen en bloc die Zustimmung zu verweigern.

Angesichts des Mangels an fachlicher Kompetenz haben die Parlamente in Lateinamerika während des 20. Jahrhunderts versucht, ihre legislative Rolle durch weitreichende Delegationen an die Exekutivgewalt und Organe der öffentlichen Verwaltung aufrechtzuerhalten. Damit wird die Reglementierungskompetenz, die in der Verfassung verankert ist, durch die direkte Übertragung gesetzgeberischer Kompetenz auf die Regierungsbehörden gleichsam noch ergänzt. Dieser scheinbar paradoxen Reaktion liegt ein verständlicher Gedankengang zugrunde: Wenn schon alle konkreten Details der Gesetzgebung von der Exekutive selbst bestimmt werden, tut man gut daran, diese Realität zu akzeptieren und dabei eindeutig klarzustellen, dass die Exekutive nur aufgrund des freiwilligen Entschlusses des Gesetzgebers über diese Ermächtigungen verfügt. Das Parlament hält auf diese Weise sein Monopol über die Gesetzgebung aufrecht, wenn auch nur auf der Ebene der Verfassungsprinzipien. In einer politischen Krise sind diese Delegationen auf jeden Fall ein starkes Machtinstrument: Werden sie vom Kongress zurückgefordert, kann eine Regierung kaum überleben.

Nun ist unmittelbar einsichtig, dass die Delegationen in einen Teufelskreis münden: Da die Exekutive die einzige Macht ist, die genaue und wirksame allgemeine Rechtsnormen setzen kann, überträgt das Parlament der Regierung immer mehr Befugnisse zur Rechtsetzung, wodurch sich die technischen Kompetenzen noch mehr als zuvor bei den Regierungsorganen konzentrieren.

Es muss aber nochmals betont werden, dass diese Art von Beziehung zwischen Gesetz und ausführender Verordnung nicht nur in Lateinamerika üblich ist. Die ausführenden Regierungsagenturen sind ein selbstverständlicher Bestandteil jeder modernen Regierung und ihre normsetzenden Befugnisse werden seit dem Klassiker „Law and Orders“ von Carleton Kemp Allen (1945) immer wieder sowohl studiert als auch (als mögliche Gefahr für die parlamentarische Macht) kritisiert.

In Lateinamerika hat sich aber die Tendenz zur Monopolisierung der normsetzenden Fachkompetenzen durch die Exekutive, wie schon oben erklärt wurde, viel weiter entwickelt und konsolidiert. Dies zeigt sich sehr deutlich an der Tatsache, dass die präsidiale Vormachtstellung bei der Rechtsetzung in der Regel entweder nicht erkannt oder nicht als problematisch angesehen wird. Dagegen werden sehr wohl die Ineffizienz und der Mangel an politischer Bedeutsamkeit des Kongresses wahrgenommen und beklagt (doch manchmal auch übertrieben). Der Zusammenhang zwischen beiden Phänomenen wird von den politi- 
schen Akteuren manchmal nicht in vollem Umfang begriffen. Dies zeigt sich nicht zuletzt an der mangelnden Entwicklung von institutionellen Mechanismen, welche die Gestaltungsmacht der Parlamente in der lateinamerikanischen Politik verstärken könnten. Wie im nächsten Punkt aufgezeigt wird, haben verschiedene derartige Mechanismen in Europa und in den USA eine lange Tradition. Es sind insgesamt drei große Modelle oder Konzepte von institutionellen Mechanismen, mit deren Hilfe die Tendenz der Exekutive zur Monopolisierung der Normsetzung konterkariert und beschränkt werden kann. Die drei Modelle sind jeweils für die USA, Großbritannien und Deutschland charakteristisch. Im nächsten Punkt werden die drei Modelle kurz dargestellt und ihre bisherige Anwendung in Lateinamerika (zutreffend für zwei der Modelle) dargelegt. Dabei geht der Autor von der Überzeugung aus, dass Experimente mit diesen Modellen (und auch mit Mischformen) in Lateinamerika unerlässlich sind, um eine Verbesserung der parlamentarischen Arbeit, insbesondere eine Verstärkung der Gestaltungsmacht der Parlamente, zu erreichen. Sonst besteht das Risiko, dass die Parlamente von einem Teil der Bürger entweder als bloße Störfaktoren oder als nutzlose und teure Körperschaften wahrgenommen werden, was für die lateinamerikanischen Demokratien nur negative Konsequenzen haben könnte.

\section{Mechanismen parlamentarischer Kontrolle der Verwaltung in den USA, Großbritannien und Deutschland sowie ihre Übertragung auf Lateinamerika}

\subsection{Independent Agencies}

Um eine Kontrolle über die Verwaltung sicher zu stellen, hat der US-amerikanische Kongress eine für dieses Land typische Verwaltungstechnik entwickelt: von der Regierung unabhängige Bundesbehörden oder Bundesausschüsse (Independent Agencies). Die erste Behörde dieser Art war die Interstate Commerce Commission, die 1887 gegründet wurde. Die Unabhängigkeit der Independent Agencies gründet insbesondere darauf, dass der Präsident ihre Mitglieder nicht aus politischen Gründen absetzen kann sondern einzig und allein aufgrund schlechter Amtsführung, Nachlässigkeit oder anderer, per Gesetz ausdrücklich geregelter Sachverhalte. Mit dem Konzept der ,unabhängigen Regierungsbehörden“ hat der Kongress die Tendenz der Exekutive zur Monopolisierung der Verwaltung, derer normsetzende Aktivitäten im 20. Jahrhundert auch in den USA enorm angewachsen sind, sehr wirkungsvoll verhindert.

Der Kongress richtet eine unabhängige Behörde ein, um ein bestimmtes Gesetz in die Praxis umzusetzen. Für die Ernennung des leitenden Personals der Behörde werden ein genaues Verfahren festgelegt und die Beschäftigungsdauer bestimmt. Des Weiteren ermächtigt das Gesetz die Behörde, Rechtsnormen zu erlassen, d.h., die Behörde verfügt über delegierte legislative Befugnisse. Dadurch, dass der Kongress diese Behörde geschaffen hat, ihr technische und administrative Autonomie verleiht sowie ihre Finanzierung 
sicherstellt, ist sie vom Präsidenten unabhängig. In gewisser Weise abhängig bleibt sie vom Kongress, indem er den Zugriff auf Information und technischen Sachverstand, über den die Behörde verfügt, behält. ${ }^{32}$

1935 erklärte das Verfassungsgericht eine Entscheidung Präsident Roosevelts für nichtig, mit der er einen von den Direktoren einer unabhängigen Kommission, der Federal Trade Commission, aus politischen Gründen hatte absetzen lassen (Humphrey's Executor $v$. United States, 295 U.S. 602-1935). Seitdem und mit Unterstützung der Judikative entwickelte sich das System der unabhängigen Behörden und wurde zu einem wesentlichen Organisationsprinzip der öffentlichen Verwaltung in den USA.

Wie wir bisher gesehen haben, stellen das Informationsdefizit hinsichtlich der öffentlichen Verwaltung und der sachlichen Details der Regierungsarbeit sowie der Mangel an qualifizierten Abgeordneten, die fähig sind, Normen für das zu regelnde Sachgebiet zu formulieren, die größten funktionellen Probleme der lateinamerikanischen Parlamente dar. Nach Ansicht des Verfassers könnten diese Probleme durch die Einrichtung von unabhängigen Behörden teilweise behoben werden. Solche unabhängigen Behörden würden es dem Kongress erlauben, auf graduelle Weise Informationen und Sachkenntnis über einen bestimmten Verwaltungsbereich zu erhalten. Es handelt sich um ein Instrument, das in bestimmten Verwaltungsbereichen experimentell angewandt werden kann.

Erste Vorläufer einer unabhängigen Behörde stellen jene parlamentarischen Organe zur finanziellen Kontrolle der Regierung dar, die in Argentinien (1994) und in Mexiko (1999) gegründet wurden. Diese Kontrollinstanzen hängen vom Parlament ab, verfügen aber über technische und administrative Autonomie. Die bloße Kontrolle der öffentlichen Ausgaben reicht jedoch nicht aus, um o.g. Wirkkraft zu erlangen, d.h. das Parlament auch mit Informations- und Sachkompetenzen hinsichtlich des Inhalts der Regierungsgeschäfte auszustatten.

In Argentinien kam es in der jüngsten Vergangenheit mit der Einrichtung der „Comisión Nacional de Evaluación y Acreditación Universitaria“ (CONEAU) zu einer sehr interessanten Erfahrung: In dieser Kommission, 1995 durch ein neues Hochschulgesetz (Gesetz Nr. 24521) ins Leben gerufen, reserviert sich das Parlament die Hauptrolle bei der Ernennung ihrer Mitglieder, die später den Präsidenten der Kommission wählen. Außerdem ist die Amtsdauer ihrer Mitglieder auf vier Jahre festgelegt worden (Art.47). from whom? Program presented by the Section of Administrative Law and Regulatory Practice American Bar Association, Administrative Law Review (Chicago), Bd. 41, Nr. 4 (Herbst 1989), S. 491-532. 
Bis heute sind die Ergebnisse dieses argentinischen „Experiments“ sehr beachtlich. Die Evaluierung von Hochschuleinrichtungen ist ein sehr komplexes Thema, über das in Argentinien bis dahin kaum Erfahrungen vorhanden waren. Dieser Kommission gelang es jedoch in wenigen Jahren klare Richtlinien und Standards auszuarbeiten und die gesamten Hochschuleinrichtungen Argentiniens in regelmäßigen Abständen zu evaluieren. Bemerkenswert ist auch ihr politischer Stil der Transparenz: So sind beispielsweise alle von ihr erlassenen Normen sowie sämtliche Evaluierungsberichte und Gutachten auf ihrer Internetseite zugänglich. Eine solche Offenheit in der Informationspolitik einer staatlichen Behörde ist in Argentinien (so wie in Lateinamerika) einmalig.

\subsection{Parlamentarische (Sonder-) Ausschüsse}

In einem parlamentarischen System fällt die Aufgabe der Regierungskontrolle vorwiegend der Opposition zu. Die Exekutive wird von der Parlamentsmehrheit gestellt, und es kann wohl kaum erwartet werden, dass sie sich selbst sehr streng kontrolliert. ${ }^{33}$ Mit dem ständigen Wachstum der öffentlichen Verwaltung im 20. Jahrhundert stieg jedoch die Sorge über die zunehmende Machtkonzentration in der Exekutive, so dass die Rolle der Opposition mit der Rolle des Parlaments als dem Verteidiger der politischen Freiheiten der Bürger identifiziert wird. ${ }^{34}$ Um eine effektive Kontrolle der Exekutive überhaupt ausüben zu können, hat jedoch die Opposition zuvörderst das Problem der Informationsbeschaffung über die Aktivitäten sowohl der Regierung als auch der Verwaltung zu bewältigen.

Das System der parlamentarischen Sonderausschüsse (Select Committees) in Großbritannien zeigt in diesem Zusammenhang eine sehr interessante Entwicklung. Die Select Committees haben sich als ein Instrument bewährt, das dem Parlament einen detaillierten Überblick über „Ausgaben, Verwaltung und Politik“ von jedem Ministerium und von jeder unabhängigen Behörde (agency) der öffentlichen Verwaltung ${ }^{35}$ ermöglicht.

Steffani bemerkt jedoch zu Recht, dass nicht nur die Opposition die parlamentarische Kontrollfunktion wahrnimmt. Das Problem ist eher, dass dies Sache der Regierungsfraktion(en) ist, und zwar in der Regel durch interne (und nicht öffentliche) Kritik. S. Winfried Steffani, Formen, Verfahren und Wirkungen der parlamentarischen Kontrolle, in: Hans-Peter Schneider / Wolfgang Zeh (Hrsg.), Parlamentsrecht und Parlamentspraxis in der Bundesrepublik Deutschland. Ein Handbuch, Berlin: Walter de Gruyter, 1989, S. 1325-1367, hier S. 1348.

Schon Max Weber vertrat die Ansicht, dass nur ein eigenständiges Enqueterecht der Opposition die Publizität der Regierungsgeschäfte garantiert. Auf diese Anregung geht die deutsche Besonderheit zurück, dass eine parlamentarische Minderheit die Einsetzung eines Untersuchungsausschusses zwingend verlangen kann. S. Max Weber, Parlament und Regierung im neugeordneten Deutschland (1. Auflage 1918), in: Johannes Winckelmann (Hrsg.), Gesammelte politische Schriften, Tübingen: Mohr, 1988, S. 306-443, hier S. 359.

In den letzten zwanzig Jahren wurde die britische öffentliche Verwaltung in vielen Bereichen durch die Einrichtung von unabhängigen Behörden (agencies) reformiert. Im Unterschied zu den 
Ende der fünfziger Jahre lieferte das Programm zur Verstaatlichung der Industrie den Anstoß zur Einführung dieses Systems. Angesichts des enormen Wachstums der öffentlichen Institutionen fürchtete man um die parlamentarischen Aufsichtsmöglichkeiten. ${ }^{36}$ So wurde ein "Sonderausschuss für die verstaatlichten Industrien" eingerichtet und mit umfangreichen Kompetenzen hinsichtlich der Kontrolle ihrer Berichte und Rechnungslegung ausgestattet. Ein zweiter Sonderausschuss, das sogenannte Estimates Committee, wurde Anfang der sechziger Jahre neu organisiert, ${ }^{37}$ so dass nun es die Aufgabe bekam, nicht nur die Ausgaben sondern auch die Effizienz der staatlichen Verwaltungsorgane zu untersuchen. Mit diesen beiden Vorläufern entwickelt sich das System in der Folge sehr schnell weiter. Bis Mitte der 70er Jahre wurden insgesamt fünf Sonderausschüsse mit sechs Unterausschüssen eingerichtet, ${ }^{38}$ die sich durch die Spezialisierung auf einem bestimmten Gebiet sowohl der Normsetzung als auch der Verwaltungsaktivität kennzeichnen. Damit beginnt die originäre und interessante Arbeitsweise der britischen Sonderausschüssen: durch die ständige Kontrolle der entsprechenden Verwaltungsstellen sichert sich das Parlament die sachlichen Informationen, die für seine Teilnahme am Prozess der Gesetzgebung unentbehrlich sind. Die „entsprechende Verwaltungsstelle“ ist in der Regel ein Ministerium, aber auch eine Unterabteilung oder eine unabhängige Behörde können das Objekt der spezialisierten Kontrolle von Sonderausschüssen sein.

Im November 1979 wurde das System der Sonderausschüsse reformiert und nach ,funktionalen“ Kriterien neu organisiert: Es wurden 14 Sonderausschüsse eingerichtet, ${ }^{39}$ die jeweils die „Ausgaben, Verwaltung und Politik“ jeder zentralen Regierungsstelle (main government departments, d.h., der wichtigsten Ministerien aber auch der ihnen untergeordneten unabhängigen Behörden) untersuchen. ${ }^{40}$ Damit konsolidierte sich die oben erwähnte

unabhängigen Behörden in den USA werden die britischen unabhängigen Behörden jedoch nicht vom Parlament durch Gesetz sondern von der Exekutive eingerichtet und sie bleiben deshalb dem entsprechenden Ministerium trotz funktionaler Autonomie hierarchisch zugeordnet. S. David Natzler / Paul Silk, Departmental Select Committees and the Next Steps Programme, in: Philip Giddings (Hrsg.), Parliamentary Accountability. A Study of Parliament and Executive Agencies, London: MacMillan, 1995, S. 71-82, hier S. 73.

Philip Giddings, op.cit. (Fn. 8), S. 669.

Es existierte seit 1945.

38 1. Expenditure Committee (eingerichtet 1971 als Nachfolger vom Estimates Committee) mit sechs Unterausschüssen gegliedert nach „,funktionalen“ Bereichen der Verwaltung a) General, b) Defence and External Affairs, c) Environment and Home Office, d) Trade and Industry, e) Education and Arts, f) Employment and Social Services. Dazu vier weitere Sonderauschüsse: 2. Science and Technology, 3. Race Relations and Immigration, 4. Overseas Development 5. Nationalised Industries.

Es sind zur Zeit sechzehn (Unterausschüsse nicht mitgerechnet).

Patrick McAuslan, Parliamentary Control of the Administrative Process, The Political Quarterly (Oxford), Bd. 60, Nr. 4 (Okt. 1989), S. 413-420, hier S. 413 und Philip Giddings, op.cit. (Fn. 34), S. 678. 
Arbeitsprozedur: Die Regierung und insbesondere die Verwaltung werden nicht nur deshalb vom Parlament überwacht und kontrolliert, weil dies eine klassische parlamentarische Aufgabe verkörpert. Die parlamentarische Kontrolle ist außerdem ein unentbehrliches Instrument der Informationsbeschaffung, denn ohne präzise und detaillierte Informationen über den Ablauf der Verwaltung könnte das Parlament überhaupt nicht am Prozess der Gesetzgebung teilnehmen. ${ }^{41}$ Besser gesagt: Ohne diese Information wäre eine sachliche Teilnahme des Parlaments am Gesetzgebungsprozess nicht möglich.

Die Select Committees werden heute als unentbehrlicher Bestandteil der Arbeit des Unterhauses angesehen. Nach Meinung von Giddings hat das System der Sonderausschüsse insbesondere drei Dinge ermöglicht: ,,[...] eine Zunahme des Umfangs der parlamentarischen Überwachung; ein Wachstum der Verantwortung gegenüber dem Parlament und eine Ausweitung der Debatte über staatliche Politik“. ${ }^{4}$

Als wichtiges Problem und Defizit des Systems wird hervorgehoben, dass die Sonderausschüsse ihre Kontrolle der Verwaltung nur nachträglich leisten können. McAuslan formuliert diese Kritik folgenderweise: ,[...] während parlamentarische Prozeduren und Strukturen reformiert und angepasst wurden, um eine bessere Untersuchung der Verwaltung sobald diese gehandelt hat - zu ermöglichen, wurden sie nicht reformiert und angepasst, um eine effektive Untersuchung derjenigen administrativen Prozesse und Systeme, die noch nicht angeführt wurden, zu leisten. “ 43

Trotz dieser Kritik kann eine Einbeziehung des Parlaments in den Verwaltungsprozess nicht als wünschenswert angesehen werden. Es sind keinesfalls die zahlreichen Entscheidungen der Verwaltung, die das Parlament rechtzeitig untersuchen sollte, damit sie eventuell verhindert werden können. Dies käme einer Einflussnahme auf dem Verwaltungsprozess gleich. Umfangreiche Informationen über den Verwaltungsprozess sind unentbehrlich, sie können jedoch m.E. ohne Kontrolldefizite nachträglich gesammelt werden. Dagegen sollten Rechtsverordnungen am besten schon vor ihrem Erlass überprüft werden, denn die Verwaltung setzt diese Normen auf Grund von legislativen Delegationen um. Das Parlament ist direkt verantwortlich für die Befugnisse, die es auf die Verwaltung übertragen hat.

Ein Gemeinsamer Sonderausschuss für Rechtsverordnungen (d.h. ein Ausschuss beider Parlamentshäuser - Joint Select Committee on Statutory Instruments) überprüft in diesem

Gesetzentwürfe werden wohlgemerkt nicht den Sonderausschüssen (select committees) sondern in der Regel den Fachausschüssen (standing committees) zur Beratung überwiesen. Die Arbeitergebnisse der Sonderausschüsse werden ständig als Berichte dem Unterhaus vorgelegt, und die meisten Sonderausschüsse verfassen mehrere Berichte pro Legislaturperiode.

Philip Giddings, op.cit. (Fn. 8), S. 680.

Patrick McAuslan, op.cit. (Fn. 38), S. 417. 
Sinne Rechtsverordnungen (statutory instruments - delegierte Legislation) ausschließlich auf ihre Übereinstimmung mit den Befugnissen, die der Verwaltung übertragen worden sind, während die anderen Sonderausschüsse eine inhaltliche Kontrolle der delegierten Legislation ausüben sollen (der Mangel an Personal, Expertise und anderen Ressourcen macht eine sehr genaue Kontrolle der delegierten Verordnungen jedoch manchmal unmöglich). Selbst McAuslan erkennt an, dass die Sonderausschüsse genug Kompetenzen zur Kontrolle der delegierten Gesetzgebung besitzen und betrachtet die Rolle des Joint Select Committee on Statutory Instruments als dermaßen wichtig, dass er die Einrichtung eines ähnlichen Ausschusses für die Überprüfung aller Gesetzentwürfe auf ihre Übereinstimmung mit den (ungeschriebenen) Verfassungsprinzipien vorschlägt. ${ }^{44}$ Problematisch ist also nicht ein Mangel an Kompetenzen sondern eher, dass der Gemeinsame Sonderausschuss für statutory instruments mit der erheblichen Anzahl von Rechtsverordnungen völlig überfordert ist. Das System hat sich jedoch deutlich bewährt, und es sollte daher (nach übereinstimmender Meinung aller Spezialisten) verstärkt werden.

Wenn wir nun die lateinamerikanischen Parlamente betrachten, erkennt man sogleich den ersten Unterschied: in Brasilien, Chile oder Argentinien beschäftigen sich die parlamentarischen Ausschüsse entweder kaum oder überhaupt nicht mit der Überwachung der ihnen evtl. entsprechenden Ministerien und Behörden. Die Ausschussarbeit wird vielmehr darin gesehen, Gesetzesentwürfe zu formulieren oder zu diskutieren. ${ }^{45}$ Und dieses Verständnis ist auch unter den Berufspolitikern und in der Öffentlichkeit sehr verbreitet: Für die wichtigste Tätigkeit der Parlamentsmitglieder hält man die Formulierung von Gesetzesentwürfen, während ihre Kontrolltätigkeiten gegenüber der Verwaltung kaum Beachtung finden. Das Ergebnis dieser einseitigen Konzeption der Parlamentsarbeit ist sehr unbefriedigend. Indem die Verwaltungsaktivität von den parlamentarischen Ausschüssen nicht kontrolliert und auch nicht regelmäßig untersucht wird, bekommen die Ausschüsse keine direkte Information über den Ablauf der Regierungsgeschäfte. Ohne diese Information ist es wiederum unmöglich, seriöse oder gut begründete Gesetzentwürfe zu konzipieren.

Das System der parlamentarischen Kommissionen hat in den verschiedenen Ländern Lateinamerikas einen unterschiedlichen Entwicklungsgrad erreicht. In Mexiko ist m.E. das System am weitesten fortgeschritten, seitdem das „Organ-Gesetz des Bundeskongresses“ (Ley Orgánica del Congreso Federal) 1999 in Kraft getreten ist. Das neue Gesetz beinhaltet wesentliche Änderungen in der parlamentarischen Arbeit. Die Anzahl der ständigen Ausschüsse wird auf 27 reduziert. Dies entspricht genau der Anzahl an Normsetzungsbefugnissen, über welche die Ministerien und Einheiten der öffentlichen Verwaltung verfügen (Paoli Bolio 2000). Die ständigen Ausschüsse des mexikanischen Parlaments sind durch

44

45

Patrick McAuslan, op.cit. (Fn. 38), S. 418.

Dieses Phänomen wird in Bezug auf Argentinien von Molinelli et al., op.cit. (Fn. 18), S. 85, beschrieben. 
die Reform in die Lage versetzt worden, eine direkte und nach Verwaltungsbereich ausdifferenzierte Kontrolle auszuüben.

In Argentinien übt das parlamentarische Kommissionssystem, wie gesagt, nur wenig oder gar keine Kontrolle über die öffentliche Verwaltung aus. ${ }^{46}$ Zur Zeit versucht der Kongress, durch die o.g. ad hoc-Kommission (Digesto Jurídico Argentino) zu den Informationen zu gelangen, die die select sommittees dem britischen Parlament zum Teil bereitstellen. Diese Kommission stellt somit einen sehr wichtigen ersten Schritt dar, ihr großes Defizit besteht jedoch in der fehlenden Verbindung zum System der parlamentarischen Ausschüsse.

\section{3 (Selbst-) Beschränkungen der Delegation: Inhalt, Zweck und Ausmaß}

Folgende Behauptung mag auf den ersten Blick paradox erscheinen: Die Delegation kann sehr wohl ein Kontrollinstrument und eine Machtressource des Parlaments sein. Diese These wird theoretisch im nächsten Punkt begründet. Wie wir sehen werden, können allerdings die legislativen Delegationen nur dann diesem Zweck konstruktiv - im Sinne der Gestaltungsmacht eines Parlaments - dienen, wenn sie präzis und mit deutlichen Grenzen formuliert werden. Dazu sind einige Richtlinien notwendig, die zu einem Leitfaden über Delegationsbeschränkungen zusammengefasst werden können. Solche Richtlinien können auch von der Justiz durchgesetzt werden, so dass beispielsweise übertriebene oder grenzenlose Delegationen für verfassungswidrig erklärt werden. Die Verfassungsgerichtsbarkeit kann hier einen wichtigen Beitrag zum Gleichgewicht zwischen Exekutive und Legislative leisten.

In diesem Sinne ist es eher ungünstig für das öffentliche Recht in Lateinamerika, dass die in dieser Region verbreitetste Lehre über Delegationsbeschränkungen vom Obersten Gerichtshof der USA stammt. Denn diese Lehre ist wenig präzis und zeigt keine praktische Wirkung. Ihr Kernpunkt bildet die Idee des intelligible principle, das die Delegation enthalten muss und das dann dem Empfänger der Delegation bei seiner normsetzenden Aktivität zu leiten hat.

Im Jahre 1935 wurden zwei Delegationen vom U.S. Supreme Court ${ }^{47}$ als verfassungswidrig erklärt, da sie nicht der Erfordernis des intelligible principle entsprachen, d.h. keine klaren Standards zur Setzung von Normen durch den Delegationsempfänger enthielten. Diese beiden Fälle sind die einzigen in der gesamten Rechtsgeschichte der USA. Seitdem gilt das 
intelligible principle-Kriterium eher als ein Interpretationskriterium und nicht als ein echtes Beschränkungskriterium. ${ }^{48}$

Für eine konkrete und eindeutige Definition legislativer Delegationen, die die Kontrollfunktion des Parlaments nicht unterlaufen können, scheint dieses Kriterium damit unzureichend zu sein. Wie schon oben angedeutet, hat das öffentliche Recht in den USA einen anderen Weg zur Kontrolle der normsetzenden Kompetenzen der Exekutive eingeschlagen. Hier werden die normsetzenden Kompetenzen nicht auf die Exekutive sondern auf unabhängige Behörden übertragen. Über diese Behörde behält der Kongress beachtliche Kontrollmöglichkeiten. Angesichts dieser ständigen parlamentarischen Kontrolle ist es politisch und rechtlich nicht so brisant, wenn eine Delegation keine klaren Grenzen oder keine genaue Bestimmungen hat: der Kongress wird die ausführende Behörde immer unter Kontrolle zu halten wissen. Aus diesem Grund ist die Lehre über Delegationsbeschränkungen in den USA eher ungenau und unwirksam geblieben, denn sie ist in der Regel nicht nötig. Der Unterschied zum öffentlichen Recht in Lateinamerika wird nun deutlich: In Lateinamerika ist es der Präsident, der die gesetzgeberischen Kompetenzen vom Kongress erhält. Übertriebene oder ungenaue Delegationen wirken sich also sehr negativ auf die Chancen des Kongresses aus, auf die Gestaltung der Politik überhaupt Einfluss zu nehmen.

Vielversprechender für den lateinamerikanischen Kontext ist m. E. die im deutschen öffentlichen Recht entwickelte Doktrin der Delegationsbeschränkung, die bisher in Lateinamerika leider kaum beachtet wurde. Im Unterschied zu den USA gilt in Deutschland die Aufhebung eines Gesetzes, das ein zuviel an Delegationen beinhaltet, durch das Verfassungsgericht als kein ungewöhnliches Geschehen, weshalb es auch mit relativer Häufigkeit geschieht. ${ }^{49}$ Die Lehre über Delegationsbeschränkung im deutschen öffentlichen Recht hat also den großen Vorteil, auf die praktische Anwendung orientiert zu sein; ihre Genauigkeit und Wirksamkeit werden angesichts der Erfahrungen der Vergangenheit, die Deutschland hinsichtlich der Gefahren zu weitreichender Delegationen gemacht hat, verständlich. ${ }^{50}$

Um die Gesetzgebungs- und Kontrollkompetenz des Parlaments sicherzustellen, bestimmt Artikel 80 Abs. 1 des deutschen Grundgesetzes: „Durch Gesetz können die Bundesregierung, ein Bundesminister oder die Landesregierungen ermächtigt werden, Rechtsverord-

50 Vor 1919 betrachtete man auch sehr weitgehende Delegationen als durchaus legitim. Während der Weimarer Republik wurde diese Praxis kritisiert, die Oberste Verfassungsbehörde, das Reichsgericht, ließ sie jedoch weiterhin zu. Es kam sogar so weit, dass die Regierung Verordnungen erlassen konnte, welche die von der Verfassung garantierten individuellen Grundrechte berühren konnten. Eines dieser Gesetze, das sog. Ermächtigungsgesetz, war es dann auch, das die Weimarer Republik beendete und zum Dritten Reich führte. Uwe Kischel, op.cit. (Fn. 3), S. 229. 
nungen zu erlassen. Dabei müssen Inhalt, Zweck und Ausmaß der erteilten Ermächtigung im Gesetze bestimmt werden. Die Rechtsgrundlage ist in der Verordnung anzugeben. Ist durch Gesetz vorgesehen, dass eine Ermächtigung weiter übertragen werden kann, so bedarf es zur Übertragung der Ermächtigung einer Rechtsverordnung“.

Ausgehend von dieser Verfassungsklausel wurde eine Vielzahl von Kriterien entwickelt, mittels derer die Delegationsbedingungen hinsichtlich der Formel „Inhalt, Zweck und Ausmaß“ festgelegt werden. Diese Kriterien unterscheiden sich nach drei Gesichtspunkten: a) die delegierte Verordnung muss für den Bürger voraussehbar sein; b) der Gesetzgeber muss selbst entscheiden, dass bestimmte Fragen geregelt werden sollen; c) das Gesetz muss ein Programm setzen, das durch die Verordnung erreicht werden soll.

Die in der Bundesrepublik Deutschlands entwickelten Kriterien von Inhalt, Zweck und Ausmaß sowie ihre praktische Anwendung sollten in Lateinamerika besser bekannt gemacht werden. Denn ein wirksamer Kriterienkatalog für die Beschränkungen der Delegation wäre von großem Vorteil, wie schon oben angedeutet, um die Delegation als eine konstruktive Machtressource der Parlamente anwenden zu können. Diese Funktion der Delegation, die von ihren Kritikern in der Regel nicht erwähnt wird, soll im nächsten Kapitel untersucht werden.

\section{Delegation als Machtressource des Parlaments}

Die argentinische Verfassungsreform versuchte zum Teil, die parlamentarische Arbeit durch Verfassungsprinzipien zu lenken. Mittels des Verbots der Delegation sollte das Parlament gewissermaßen dazu gezwungen werden, seine Fähigkeit zur Gestaltung der Politik und der Gesetzgebung zu erweitern. Das Verbot der Delegation in Argentinien hat sich jedoch aus zwei Gründen als wenig realistisch erwiesen: erstens verfügt das argentinische Parlament nicht über die technische Kapazität, alle übertragene Kompetenzen zur Rechtsetzung wieder zu übernehmen, und zweitens ist eine moderne Verwaltung ohne die Übertragung rechtssetzender Kompetenzen nicht mehr denkbar.

Die Koexistenz von Delegation und Reglementierungskompetenz in den verschiedenen lateinamerikanischen Ländern wurde oben schon erwähnt. In beiden Fällen geht es um Rechtsverordnungen, die ein Gesetz durch verschiedene Präzisierungen oder andere Bestimmungen ergänzen sollen. Bei der Reglementierungskompetenz erlässt die Exekutive solche Verordnungen völlig unabhängig von einer parlamentarischen Ermächtigung, denn die Reglementierungskompetenz ist ja in der Verfassung verankert. Im Falle der Delegation wird die Exekutive vom Parlament ausdrücklich dazu ermächtigt, Rechtsverordnungen zu einem Gesetz zu erlassen. Es gibt ansonsten keine genaue Unterschiede zwischen beiden Instrumenten (Die Reglementierungskompetenz wird daher manchmal als eine ,uneigentli- 
che Delegation“ charakterisiert), eine Unklarheit, die von den lateinamerikanischen Parlamenten zur Entwicklung einer besonderen, schon vorher erwähnten Strategie benutzt werden kann: durch ausdrückliche Delegation von rechtsetzenden Kompetenzen wird die autonome Ausübung der präsidialen Reglementierungskompetenz tendenziell verhindert. Eine ausdrückliche Delegation ist auf jeden Fall eine klare Rechtsgrundlage zum Erlass von Dekreten oder Verordnungen, denn der Kongress kann das Ausmaß jeder einzelnen Delegation präzis festsetzen. Die Grenzen der Reglementierungskompetenz sind dagegen unklar: die lateinamerikanischen Verfassungen benutzen hier Formulierungen wie den „Geist des Gesetzes“ oder ähnliche, um die Grenzen der präsidialen Reglementierungskompetenz festzulegen. Es kann also unter Umständen im Interesse der Exekutive sein, ihre Rechtsverordnungen auf der Basis einer präzisen Delegation zu erlassen. Die Gültigkeit eines Reglementierungsdekrets kann nämlich immer vor Gericht bestritten werden, wenn das Dekret dem „Geist des Gesetzes“ widerspricht. Ob ein Reglementierungsdekret von einem Gericht bestätigt wird, ist also eine ziemlich willkürliche Frage. Nicht so bei einer Delegation mit deutlichem Inhalt, Zweck und Ausmaß. Deswegen kann es für die Exekutive auf jeden Fall günstiger sein, ihre Normsetzung auf eine Delegation statt auf die Reglementierungskompetenz zu begründen. Wenn ein Gesetz übrigens eine klare Delegation beinhaltet, wird die Exekutive in gewisser Hinsicht sowieso vom Gebrauch ihrer Reglementierungskompetenz abgehalten. Denn eine präzise Delegation überträgt auf die Exekutive genug Kompetenzen zur Rechtssetzung, die eine zusätzliche Reglementierung des Gesetzes überflüssig machen.

Ob die Exekutive von der Reglementierungskompetenz Gebrauch macht oder nicht, ist natürlich zum Teil eine politische Frage. Die Delegation bietet jedoch ein Instrument an, durch dessen geschickte Anwendung das Parlament die rechtsetzenden Kompetenzen der Exekutive kontrollieren und beschränken kann. Jedes Parlament in Lateinamerika sollte sich langfristig das politische Ziel setzten, dass rechtssetzende Kompetenzen vom Präsidenten nur mit Genehmigung (am besten durch Delegation) des Parlaments ausgeübt werden. In diesem Sinne könnte die präzise und reguläre Anwendung der Delegation, zusammen mit den anderen in diesem Artikel diskutierten Instrumenten, für lateinamerikanische Parlamente eine wirksame Machtressource sein, um die rechtssetzenden Aktivitäten der Regierung und der Verwaltung unter Kontrolle zu halten. Denn nur durch diese Kontrolle schafft sich ein Parlament in unseren Tagen die nötige Information und Fachkompetenz, die ihm überhaupt erst ermöglichen, seine ursprüngliche Rolle als Gesetzgeber wahrzunehmen. 